\title{
Cohomology Theory of Nonassociative Algebras with Metagroup Relations
}

\author{
Sergey V. Ludkowski \\ Department of Applied Mathematics, MIREA—Russian Technological University, av. Vernadsky 78, \\ 119454 Moscow, Russia; sludkowski@mail.ru
}

Received: 30 April 2019; Accepted: 24 June 2019; Published: 4 July 2019

\begin{abstract}
Nonassociative algebras with metagroup relations and their modules are studied. Their cohomology theory is scrutinized. Extensions and cleftings of these algebras are studied. Broad families of such algebras and their acyclic complexes are described. For this purpose, different types of products of metagroups are investigated. Necessary structural properties of metagroups are studied. Examples are given. It is shown that a class of nonassociative algebras with metagroup relations contains a subclass of generalized Cayley-Dickson algebras.
\end{abstract}

Keywords: nonassociative algebra; cohomology; extension; metagroup

MSC: 16E40; 16D70; 18G60; 17A60; 03C60; 03C90

\section{Introduction}

Nonassociative algebras comprise a large area of algebra. Among them, Lie algebras and their modifications are widely used in different branches of mathematics and its applications including PDEs, physics, quantum mechanics, informatics, and biology (see, for example, [1-5] and references therein). There are other classes of nonassociative algebras which are less investigated. For example, octonions and generalized Cayley-Dickson algebras play very important roles in mathematics and quantum field theory [6-11]. Their structures and identities have attracted great attention. They are used not only in algebra and noncommutative geometry, but also in noncommutative analysis and PDEs, particle physics, mathematical physics, in the theory of Lie groups and algebras and their generalizations, mathematical analysis, operator theory, and in applications in natural sciences including physics and quantum field theory (see [2,7-9,12-25] and references therein).

A multiplicative law of their canonical generators is nonassociative and leads to a more general notion of a metagroup instead of a group [26]. The preposition meta is used to emphasize that such an algebraic object has milder properties than a group. Their axiomatic metagroups satisfy Conditions (1)-(3) with the weak relation (9), as shown in Definition 1 in Section 2. They were used in [26] to investigate automorphisms and derivations of nonassociative algebras.

An extensive area of investigation of PDEs intersects with cohomologies and deformed cohomologies [27]. Therefore, it is important to develop this area using octonions, Cayley-Dickson algebras, and more general metagroup algebras.

It appears that generators of Cayley-Dickson algebras form objects, which are nonassociative generalizations of groups. They are called metagroups. This means that metagroup algebras include the Cayley-Dickson algebras . This article is devoted to algebras generated by metagroups. Note that a class of metagroups differs substantially from a class of groups. Indeed, a metagroup may be nonassociative, power non-associative, or nonalternative. Moreover, left or right inverse elements in the metagroup may not exist or it may contain elements for which left and right inverse elements do not coincide (see Definition 1 in Section 2). 
On the other hand, algebras are frequently studied using cohomology theory. However, the already developed cohomology theory operates with associative algebras. It has been investigated by Hochschild and other authors [1,28-30], but it is not applicable to nonassociative algebras. In some particular cases of nonassociative algebras, such as Lie algebras, pre-Lie algebras, flexible algebras, and alternative algebras, homology theory was developed for the needs of studies of their structures (see, for example, [2,31-33] and references therein). It is necessary to note that classes of these algebras are quite different from classes of generalized Cayley-Dickson algebras and nonassociative algebras with metagroup relations. This work is devoted to the development of cohomology theory for nonassociative algebras, namely for its subclass of algebras with metagroup relations.

Previously, cohomologies of loop spaces on quaternion and octonion manifolds were studied in [17]. They have specific features in comparison with the case of complex manifolds. This is especially caused by the noncommutativity of the quaternion skew field and the nonassociativity of the octonion algebra.

In this article, nonassociative algebras with metagroup relations are studied. Their modules and acyclic complexes are investigated. Their cohomology theory is scrutinized in Section 2 . This requires the development of a specific axiomatic model of such algebras and their modules. Necessary structural properties of metagroups are studied in Lemmas 1 and 2. Acyclic complexes and co-chain complexes are described in Proposition 1 and Theorem 1. A relation of the cohomologies with quotient modules is given by Theorem 2. Extensions and cleftings of these algebras are studied in Theorems 3-5 under the framework of cohomology theory. Broad families of such algebras are described. In Theorem 6, inner derivations of nonassociative algebras are investigated. A semisimplicity of nonassociative algebras is investigated in Theorem 7 and Corollary 1.

Different types of products of metagroups are investigated in Theorems 8 and 9 in Section 3. Examples are given. It is shown that a class of nonassociative algebras with metagroup relations contains a subclass of generalized Cayley-Dickson algebras.

All of the key results of this paper are obtained for the first time. They can be used for further studies of nonassociative algebras cohomologies, the structure of nonassociative algebras, operator theory, and the spectral theory of Cayley-Dickson algebras, PDEs, noncommutative analysis, noncommutative geometry, mathematical physics, and their applications in the sciences.

\section{Cohomology Theory of Nonassociative Algebras}

To avoid misunderstandings we give the necessary definitions.

Definition 1. Let $G$ be a set with a single-valued binary operation (multiplication) $G^{2} \ni(a, b) \mapsto a b \in G$, where $G$ satisfies the following conditions:

(1) For each $a$ and $b$ in $G$, there is a unique $x \in G$ with $a x=b$ and

(2) A unique $y \in G$ exists, satisfying $y a=b$, which is denoted by $x=a \backslash b=\operatorname{Div}_{l}(a, b)$ and $y=b / a=$ $\operatorname{Div}_{r}(a, b)$ correspondingly,

(3) A neutral (i.e., unit) element $e_{G}=e \in G$ exists: $e g=g e=g$ for each $g \in G$.

The set of all elements $h \in G$ commuting and associating with $G$ is

(4) $\operatorname{Com}(G):=\{a \in G: \forall b \in G, a b=b a\}$,

(5) $N_{l}(G):=\{a \in G: \forall b \in G, \forall c \in G,(a b) c=a(b c)\}$,

(6) $N_{m}(G):=\{a \in G: \forall b \in G, \forall c \in G,(b a) c=b(a c)\}$,

(7) $N_{r}(G):=\{a \in G: \forall b \in G, \forall c \in G,(b c) a=b(c a)\}$,

(8) $N(G):=N_{l}(G) \cap N_{m}(G) \cap N_{r}(G)$;

$\mathcal{C}(G):=\operatorname{Com}(G) \cap N(G)$ is called the center $\mathcal{C}(G)$ of $G$.

We call $G$ a metagroup if a set $G$ possesses a single-valued binary operation and satisfies Conditions (1)-(3) and 
(9) $(a b) c=\mathrm{t}_{3}(a, b, c) a(b c)$

for each $a, b$, and $c$ in $G$, where $t_{3}(a, b, c) \in \mathbf{\Psi}, \mathbf{\Psi} \subset \mathcal{C}(G)$;

where $t_{3}$ shortens a notation $t_{3, G}$, where $\Psi$ denotes a (proper or improper) subgroup of $\mathcal{C}(G)$.

Then $G$ will be called a central metagroup if, in addition to (9), it satisfies the condition

(10) $a b=t_{2}(a, b) b a$

for each $a$ and $b$ in $G$, where $\mathrm{t}_{2}(a, b) \in \mathbf{\Psi}$.

Particularly, $\operatorname{Inv}_{l}(a)=\operatorname{Div}_{l}(a, e)$ is a left inversion, and $\operatorname{Inv}_{r}(a)=\operatorname{Div}_{r}(a, e)$ is a right inversion.

In view of the nonassociativity of $G$, in general, a product of several elements of $G$ is usually specified by opening "(" and closing ")" parentheses. We denote the product of elements $a_{1}, \ldots, a_{n}$ in $G$ by $\left\{a_{1}, \ldots, a_{n}\right\}_{q(n)}$, where a vector $q(n)$ indicates an order of pairwise multiplications of elements in the row $a_{1}, \ldots, a_{n}$ in braces in the following manner. The enumerate positions are as follows: before $a_{1}$ by 1 , between $a_{1}$ and $a_{2}$ by $2, \ldots$, by $n$ between $a_{n-1}$ and $a_{n}$, and by $n+1$ after $a_{n}$. Then, we put $q_{j}(n)=(k, m)$ if there are $k$ opening "(" and $m$ closing ")" parentheses in the ordered product at the $j$-th position of the type $). .).\left(\ldots\left(\right.\right.$, where $k$ and $m$ are nonnegative integers, $q(n)=\left(q_{1}(n), \ldots ., q_{n+1}(n)\right)$ with $q_{1}(n)=(k, 0)$ and $q_{n+1}(n)=(0, m)$.

Traditionally, $S_{n}$ denotes the symmetric group of the set $\{1,2, \ldots, n\}$. Henceforth, maps and functions on metagroups are assumed to be single-valued unless otherwise specified .

Let $\psi: G \rightarrow G$ be a bijective surjective map satisfying the following condition: $\psi(a b)=\psi(a) \psi(b)$ for each $a$ and $b$ in $G$. Then, $\psi$ is called an automorphism of the metagroup $G$.

Lemma 1. (i). Let $G$ be a central metagroup. Then, for every $a_{1}, \ldots, a_{n}$ in $G, v \in S_{n}$ and vectors $q(n)$ and $u(n)$ indicating an order of pairwise multiplications and $n \in \mathbf{N}$, there exists an element $t_{n}=t_{n}\left(a_{1}, \ldots, a_{n} ; q(n), u(n) \mid v\right) \in \mathbf{\Psi}$ such that

$$
\left\{a_{1}, \ldots, a_{n}\right\}_{q(n)}=t_{n}\left\{a_{v(1)}, \ldots, a_{v(n)}\right\}_{u(n)} .
$$

(ii). If $G$ is a metagroup and if $v$ is the neutral element $v=$ id in $S_{n}$, then property (1) is satisfied.

Proof. From Conditions (1)-(8) in Definition 1, it follows that $\mathcal{C}(G)$ itself is a commutative group.

(i). For $n=1$, evidently $t_{1}=1$, since $a=1 a$ for each $a \in G$. For $n=2$, Formula (1) is a direct consequence of condition (10) in Definition 1 . Consider $n=3$. When $u$ is the identity element of $S_{3}$, the statement follows from condition (9) in Definition 1. For any transposition $u$ of two elements of the set $\{1,2,3\}$, the statement follows from (9) and (10) in Definition 1. Elements of $S_{3}$ can be obtained by multiplication of pairwise transpositions. Therefore, from the condition $\Psi \subset \mathcal{C}(G)$, it follows that formula (1) is valid.

Now, let $n \geq 4$ and suppose that this lemma is proved for any products consisting of less than $n$ elements. In view of Properties (1) and (2) in Definition 1, it is sufficient to verify Formula (1) of this lemma for $\left\{a_{1}, \ldots, a_{n}\right\}_{q(n)}=\left(\ldots\left(\left(a_{1} a_{2}\right) a_{3}\right) \ldots\right) a_{n}=:\left\{a_{1}, \ldots, a_{n}\right\}_{l(n)}$ since $\mathbf{\Psi} \subset$ $\mathcal{C}(G)$. In this particular case, $\left\{a_{v(1)}, \ldots, a_{v(n)}\right\}_{u(n)}=\left\{a_{v(1)}, \ldots, a_{v(n-1)}\right\}_{u(n-1)} a_{n}$. Formula (1) follows from the induction hypothesis, since $\left(\ldots\left(\left(a_{1} a_{2}\right) a_{3}\right) \ldots\right) a_{n-1}=t_{n-1}\left\{a_{v(1)}, \ldots, a_{v(n-1)}\right\}_{u(n-1)}$ and hence $\left(\left(\ldots\left(\left(a_{1} a_{2}\right) a_{3}\right) \ldots\right) a_{n-1}\right) a_{n}=t_{n-1}\left(\left\{a_{v(1)}, \ldots, a_{v(n-1)}\right\}_{u(n-1)} a_{n}\right)$ and putting $t_{n}=t_{n-1}$, where $t_{n-1}=$ $t_{n-1}\left(a_{1}, \ldots, a_{n-1} ; q(n-1), u(n-1) \mid w\right)$ with $w=\left.v\right|_{\{1, \ldots, n-1\}}, v(n)=n$.

In the general case, $\left\{a_{v(1)}, \ldots, a_{v(n)}\right\}_{u(n)}=\left\{b_{1}, \ldots, b_{j}, \ldots, b_{k}\right\}_{p(k)}$, where $j$ is such that either $b_{j}=c_{j} a_{n}$ with $c_{j}=\left\{a_{v(j)}, \ldots, a_{v(j+m-1)}\right\}_{r(m)}$ and with $v(j+m)=n$ or $b_{j}=a_{n} c_{j}$ with $c_{j}=\left\{a_{v(j+1)}, \ldots, a_{v(j+m)}\right\}_{r(m)}$ and with $v(j)=n$. Also, $b_{1}=a_{v(1)}, \ldots, b_{j-1}=a_{v(j-1)}, b_{j+1}=a_{v(j+1)}, \ldots, b_{k}=a_{v(n)}$ with suitable vectors $p(k)$ and $r(m)$. If $m>1$, then $k<n$ and using the induction hypothesis for $\left\{b_{1}, \ldots, b_{j}, \ldots, b_{k}\right\}_{p(k)}$ and $b_{j}$, we get that elements $s$ and $t$ in $\Psi$ exist so that $\left\{b_{1}, \ldots, b_{j}, \ldots, b_{k}\right\}_{p(k)}=s\left\{b_{1}, \ldots, b_{j-1}, b_{j+1}, \ldots, b_{k}\right\}_{p(k-1)} b_{j}$ $=s t\left(\left\{b_{1}, \ldots, b_{j-1}, b_{j+1}, \ldots, b_{k}\right\}_{p(k-1)} c_{j}\right) a_{n}$, where $p(k-1)$ is a corresponding vector prescribing an order of multiplications. 
Again, applying the induction hypothesis to the product of $n-1$ elements $\left\{b_{1}, \ldots, b_{j-1}, b_{j+1}, \ldots, b_{k}\right\}_{p(k-1)} c_{j}$, we deduce that there exists $w \in \mathbf{\Psi}$, such that

$\left\{a_{v(1)}, \ldots, a_{v(n)}\right\}_{u(n)}=\operatorname{stw}\left(\left(\ldots\left(\left(a_{1} a_{2}\right) a_{3}\right) \ldots\right) a_{n-1}\right) a_{n}$.

Therefore, a case remains when $m=1$. Let the first multiplication in $\left\{a_{v(1)}, \ldots, a_{v(n)}\right\}_{u(n)}$ containing $a_{n}$ be $\left(a_{v(k)} a_{v(k+1)}\right)=: b_{k}$. We put $b_{j}=a_{v(j)}$ for each $1 \leq j \leq k-1$. We also put $b_{j-1}=a_{v(j)}$ for each $k+1<j \leq n$, where either $a_{n}=a_{v(k)}$ or $a_{n}=a_{v(k+1)}$. Therefore, using previous identities, we rewrite the considered product as $\left\{a_{v(1)}, \ldots, a_{v(n)}\right\}_{u(n)}=\left\{b_{y(1)}, \ldots, b_{y(n-1)}\right\}_{w(n-1)}$ with an element $y \in S_{n-1}$ of the symmetric group and a vector $w(n-1)$, indicating an order of pairwise products (see Definition 1). From the induction hypothesis, we deduce that there exists $t_{n-1} \in \mathbf{\Psi}$, so that $t_{n-1}\left\{b_{y(1)}, \ldots, b_{y(n-1)}\right\}_{w(n-1)}=p b_{k}$ with $p=\left\{b_{1}, \ldots, b_{k-1}, b_{k+1}, \ldots, b_{n-1}\right\}_{w(n-1)}$, because $G$ is the central metagroup. Applying the induction hypothesis for $n=3$, we infer that $t_{3} \in \boldsymbol{\Psi}$ exists, such that $t_{n-1} t_{3}\left\{b_{y(1)}, \ldots, b_{y(n-1)}\right\}_{w(n-1)}=(p a) a_{n}$, where either $a=a_{v(k+1)}$ or $a=a_{v(k)}$, correspondingly. From the induction hypothesis for $n-1$, it follows that $\tilde{t}_{n-1} \in \mathbf{\Psi}$ exists, so that $\tilde{t}_{n-1} p a=\left(\ldots\left(\left(a_{1} a_{2}\right) a_{3}\right) \ldots\right) a_{n-1}$, and hence, $\left\{a_{1}, \ldots, a_{n}\right\}_{l(n)}=t_{n}\left\{a_{v(1)}, \ldots, a_{v(n)}\right\}_{u(n)}$, where $t_{n}=\tilde{t}_{n-1} t_{n-1} t_{3}$.

(ii). Now, let $G$ be a metagroup and $v=i d$ be the neutral element of the symmetric group $S_{n}$, where $i d(k)=k$ for each $k \in \mathbf{N}$. Then, using condition (10) of Definition 1 is unnecessary, because transpositions are already not utilized. For $n=1$ and $n=2$, we get $t_{1}=1$ and $t_{2}=1$, since $a=1 a$ and $a b=1 a b$ for each $a$ and $b$ in $G$. For $n=3$, Formula (1) of this lemma follows on from condition (9) in Definition 1. Then, the proof in case (ii) by induction is a simplification of that of case $(i)$.

Lemma 2. If $G$ is a metagroup, then for each $a$ and $b \in G$, the following identities are fulfilled:

(1) $b \backslash e=(e / b) \mathrm{t}_{3}(e / b, b, b \backslash e)$;

(2) $(a \backslash e) b=(a \backslash b) \mathrm{t}_{3}(e / a, a, a \backslash e) / \mathrm{t}_{3}(e / a, a, a \backslash b)$;

(3) $b(e / a)=(b / a) \mathrm{t}_{3}(b / a, a, a \backslash e) / \mathrm{t}_{3}(e / a, a, a \backslash e)$.

Proof. Conditions (1)-(3) in Definition 1 imply that

(4) $b(b \backslash a)=a, b \backslash(b a)=a$;

(5) $(a / b) b=a,(a b) / b=a$

for each $a$ and $b$ in G. Using Condition (9) in Definition 1 and Identities (4) and (5), we deduce that $e / b=(e / b)(b(b \backslash e))=(b \backslash e) / \mathrm{t}_{3}(e / b, b, b \backslash e)$ which leads to $(1)$.

Let $c=a \backslash b$. Then, from Identities (1) and (4), it follows that $(a \backslash e) b=(e / a) \mathrm{t}_{3}(e / a, a, a \backslash e)(a c)$ $=((e / a) a)(a \backslash b) \mathrm{t}_{3}(e / a, a, a \backslash e) / \mathrm{t}_{3}(e / a, a, a \backslash b)$ which provides (2).

Now, let $d=b / a$. Then, Identities (1) and (5) imply that $b(e / a)=(d a)(a \backslash e) / \mathrm{t}_{3}(e / a, a, a \backslash e)=$ $(b / a) \mathrm{t}_{3}(b / a, a, a \backslash e) / \mathrm{t}_{3}(e / a, a, a \backslash e)$ which demonstrates (3).

Definition 2. Let $A$ be an algebra over an associative unital ring $\mathcal{T}$, such that $A$ has a natural structure of $a(\mathcal{T}, \mathcal{T})$-bimodule with a multiplication map $A \times A \rightarrow A$, which is right and left distributive, $a(b+c)=$ $a b+a c,(b+c) a=b a+c a$, and also satisfies the identities $r(a b)=(r a) b,(a r) b=a(r b),(a b) r=a(b r)$, $s(r a)=(s r) a$, and $(a r) s=a(r s)$ for any $a, b$, and $c$ in $A, r$ and $s$ in $\mathcal{T}$. Let $G$ be a metagroup and $\mathcal{T}$ be an associative unital ring.

Henceforth, the ring $\mathcal{T}$ is assumed to be commutative, unless otherwise specified.

Then, by $\mathcal{T}[G]$, a metagroup algebra is denoted over $\mathcal{T}$ for all formal sums $s_{1} a_{1}+\ldots+s_{n} a_{n}$ satisfying Conditions $(1-3)$ below, where $n$ is a positive integer, $s_{1}, \ldots, s_{n}$ are in $\mathcal{T}$, and $a_{1}, \ldots, a_{n}$ belong to $G$ :

(1) $s a=$ as for each $s$ in $\mathcal{T}$ and $a$ in $G$,

(2) $s(r a)=(s r)$ a for each $s$ and $r$ in $\mathcal{T}$, and $a \in G$,

(3) $r(a b)=(r a) b,(a r) b=a(r b),(a b) r=a(b r)$ for each $a$ and $b$ in $G, r \in \mathcal{T}$. 
Note 1. Let $M$ be an additive commutative group such that $M$ is a two-sided $G$-module, where $G$ is a metagroup. We remind the reader that this means that automorphisms $p(g)$ and $s(g)$ of $M$ correspond to each $g \in G$. For short, we use $g x=p(g) x$ and $x g=x s(g)$ for each $g \in G$.

Note that, usually, $M$ has a natural structure of a two-sided Z-module, because $M$ is the additive commutative group, where $\mathbf{Z}$ denotes the ring of all integers. Therefore, $M$ is a two-sided G-module if and only if it is a two-sided $\mathbf{Z}[G]$-module according to the formulas $\left(\sum_{g \in G} n(g) g\right) x=\sum_{g \in G} n(g)(g x)$ and $x\left(\sum_{g \in G} n(g) g\right)=\sum_{g \in G}(x g) n(g)$, where $n(g) \in \mathbf{Z}$ for each $g \in G$.

One can consider the additive group of integers $\mathbf{Z}$ as the trivial two-sided G-module putting $g n=n g=n$ for each $g \in G$ and $n \in \mathbf{Z}$, where $G$ is a metagroup.

Example 1. I. Recall the following: Let $A$ be a unital algebra over a commutative associative unital ring $F$ supplied with a scalar involution $a \mapsto \bar{a}$ so that its norm $N$ and trace $T$ maps have values in $F$ and fulfill conditions:

(1) $a \bar{a}=N(a) 1$ with $N(a) \in F$,

(2) $a+\bar{a}=T(a) 1$ with $T(a) \in F$,

(3) $T(a b)=T(b a)$

for each $a$ and $b$ in $A$.

If a scalar $f \in F$ satisfies the condition $\forall a \in A f a=0 \Rightarrow a=0$, then such element $f$ is called cancelable. For a cancelable scalar $f$, the Cayley-Dickson doubling procedure provides new algebra $C(A, f)$ over $F$ such that

(4) $C(A, f)=A \oplus A l$,

(5) $(a+b l)(c+d l)=(a c-f \bar{d} b)+(d a+b \bar{c}) l$ and

(6) $\overline{(a+b l)}=\bar{a}-b l$

for each $a$ and $b$ in $A$. Then, $l$ is called a doubling generator. From the definitions of $T$ and $N$, it follows that $\forall a \in A, \forall b \in A T(a)=T(a+b l)$ and $N(a+b l)=N(a)+f N(b)$. The algebra $A$ is embedded into $C(A, f)$ as $A \ni a \mapsto(a, 0)$, where $(a, b)=a+b l$. This is put by induction $A_{n}\left(f_{(n)}\right)=C\left(A_{n-1}, f_{n}\right)$, where $A_{0}=A$, $f_{1}=f, n=1,2, \ldots, f_{(n)}=\left(f_{1}, \ldots, f_{n}\right)$. Then, $A_{n}\left(f_{(n)}\right)$ are generalized Cayley-Dickson algebras when $F$ is not a field or Cayley-Dickson algebras when $F$ is a field.

It is natural to put $A_{\infty}(f):=\bigcup_{n=1}^{\infty} A_{n}\left(f_{(n)}\right)$, where $f=\left(f_{n}: n \in \mathbf{N}\right)$. If $\operatorname{char}(F) \neq 2$, let $\operatorname{Im}(z)=$ $z-T(z) / 2$ be the imaginary part of a Cayley-Dickson number $z$, and hence $N(a):=N_{2}(a, \bar{a}) / 2$, where $N_{2}(a, b):=T(a \bar{b})$.

If the doubling procedure starts from $A=F 1=: A_{0}$, then $A_{1}=C\left(A, f_{1}\right)$ is a $*$-extension of $F$. If $A_{1}$ has a basis $\{1, u\}$ over $F$ with the multiplication table $u^{2}=u+w$, where $w \in F$ and $4 w+1 \neq 0$ with the involution $\overline{1}=1, \bar{u}=1-u$, then $A_{2}$ is the generalized quaternion algebra, and $A_{3}$ is the generalized octonion (Cayley-Dickson) algebra.

When $F=\mathbf{R}$ and $f_{n}=1$, each $n$ by $\mathcal{A}_{r}$ will denote the real Cayley-Dickson algebra with generators $i_{0}, \ldots, i_{2^{r}-1}$, such that $i_{0}=1, i_{j}^{2}=-1$ for each $j \geq 1, i_{j} i_{k}=-i_{k} i_{j}$ for each $j \neq k \geq 1$. Note that the Cayley-Dickson algebra $\mathcal{A}_{r}$ for each $r \geq 3$ is nonassociative, for example, $\left(i_{1} i_{2}\right) i_{4}=-i_{1}\left(i_{2} i_{4}\right)$, etc. Moreover, for each $r \geq 4$, the Cayley-Dickson algebra $\mathcal{A}_{r}$ is nonalternative (see [7-9]). Frequently, $\bar{a}$ is also denoted by $a^{*}$ or $\tilde{a}$. Then, $G_{r}=\left\{i_{j},-i_{j}: j=0,1, \ldots, 2^{r}-1\right\}$ is a finite metagroup for each $3 \leq r<\infty$.

Let $A_{n}$ be a Cayley-Dickson algebra over a commutative associative unital ring $\mathcal{R}$ that is characteristically different from two, such that $A_{0}=\mathcal{R}, n \geq 2$. Take its basic generators $i_{0}, i_{1}, \ldots, i_{2^{n}-1}$, where $i_{0}=1$. Choose $\Psi$ as a multiplicative subgroup contained in the ring $\mathcal{R}$, such that $f_{j} \in \Psi$ for each $j=0, \ldots, n$. Put $G_{n}=\left\{i_{0}, i_{1}, \ldots, i_{2^{n}-1}\right\} \times \mathbf{\Psi}$. Then, $G_{n}$ is a central metagroup.

II. More generally, let $H$ be a group such that $\Psi \subset H$ with relations $h i_{k}=i_{k} h$ and $(h g) i_{k}=h\left(g i_{k}\right)$ for each $k=0,1, \ldots, 2^{n}-1$ and each $h$ and $g$ in $H$. Then, $G_{n}=\left\{i_{0}, i_{1}, \ldots, i_{2^{n}-1}\right\} \times H$ is also a metagroup. If the group $H$ is noncommutative, then the latter metagroup can be noncentral (see Condition (10) in 
Definition 1). Using the notation of Example 1. I, we get that the Cayley-Dickson algebra $\mathcal{A}_{\infty}$ over the real field $\mathbf{R}$ with $f_{n}=1$ for each $n$ provides an example of a metagroup $G_{\infty}=\left\{i_{j},-i_{j}: 0 \leq j \in \mathbf{Z}\right\}$, where $\mathbf{Z}$ denotes the ring of integers.

III. Generally metagroups need not be central. From given metagroups, new metagroups can be constructed using their direct or semidirect products. Certainly, each group is a metagroup also. Therefore, there are abundant families of noncentral metagroups and also of central metagroups different from groups.

In another way, smashed products of groups and of metagroups can be considered by providing other examples of metagroups (for more detail, see Section 3).

Definition 3. Let $\mathcal{R}$ be a ring, which may be nonassociative relative to the multiplication. If the mapping $\mathcal{R} \times M \rightarrow M, \mathcal{R} \times M \ni(a, m) \mapsto a m \in M$ exists, such that $a(m+k)=a m+a k$ and $(a+b) m=a m+b m$ for each $a$ and $b$ in $\mathcal{R}, m$ and $k$ in $M$, then $M$ will be called a generalized left $\mathcal{R}$-module or, for short, $a$ left $\mathcal{R}$-module or left module over $\mathcal{R}$.

If $\mathcal{R}$ is a unital ring and $1 m=m$ for each $m \in M$, then $M$ is called a left unital module over $\mathcal{R}$, where 1 denotes the unit element in the ring $\mathcal{R}$. Symmetrically, a right $\mathcal{R}$-module is defined.

If $M$ is a left and right $\mathcal{R}$-module, then it is called a two-sided $\mathcal{R}$-module or a $(\mathcal{R}, \mathcal{R})$-bimodule. If $M$ is a left $\mathcal{R}$-module and a right $\mathcal{S}$-module, then it is called a $(\mathcal{R}, \mathcal{S})$-bimodule.

A two-sided module $M$ over $\mathcal{R}$ is called cyclic if an element $y \in M$ exists such that $M=\mathcal{R}(y \mathcal{R})$ and $M=(\mathcal{R} y) \mathcal{R}$, where $\mathcal{R}(y \mathcal{R})=\{s(y p): s, p \in \mathcal{R}\}$ and $(\mathcal{R} y) \mathcal{R}=\{(s y) p: s, p \in \mathcal{R}\}$.

Let $G$ be a metagroup. Take a metagroup algebra $A=\mathcal{T}[G]$ and a two-sided $A$-module $M$, where $\mathcal{T}$ is an associative unital ring (see Definition 2). Let $M_{g}$ be a two-sided $\mathcal{T}$-module for each $g \in G$, where $G$ is the metagroup. Let $M$ have the decomposition $M=\sum_{g \in G} M_{g}$ as a two-sided $\mathcal{T}$-module. Let $M$ also satisfy the following conditions:

(1) $h M_{g}=M_{h g}$ and $M_{g} h=M_{g h}$

(2) $(b h) x_{g}=b\left(h x_{g}\right)$ and $x_{g}(b h)=\left(x_{g} h\right) b$ and $b x_{g}=x_{g} b$,

(3) (hs) $x_{g}=\mathrm{t}_{3}(h, s, g) h\left(s x_{g}\right)$ and $\left(h x_{g}\right) s=\mathrm{t}_{3}(h, g, s) h\left(x_{g} s\right)$ and $\left(x_{g} h\right) s=\mathrm{t}_{3}(g, h, s) x_{g}(h s)$

for every $h, g, s$ in $G$ and $b \in \mathcal{T}$ and $x_{g} \in M_{g}$. Then, a two-sided $A$-module $M$ satisfying conditions (1)-(3) is called smashly G-graded. For short, it is also called "G-graded" instead of "smashly G-graded". In particular, if the module $M$ is G-graded and splits into a direct sum $M=\bigoplus_{g \in G} M_{g}$ of two-sided $\mathcal{T}$-submodules $M_{g}$, then we say that that $M$ is directly $G$-graded. For a nontrivial (nonzero) $G$-graded module $X$ with the nontrivial metagroup $G$, it is supposed that $g \in G$ exists such that $X_{g} \neq X_{e}$ if something else is not outlined.

Similarly, G-graded left and right $A$-modules are defined. Henceforward, speaking about $A$-modules (left, right, or two-sided), it is supposed that they are G-graded and, for short, "an $A$-module" is written instead of "a G-graded $A$-module", unless otherwise specified.

If $P$ and $N$ are left $A$-modules and a homomorphism $\gamma: P \rightarrow N$ is such that $\gamma(a x)=a \gamma(x)$ for each $a \in A$ and $x \in P$, then $\gamma$ is called a left $A$-homomorphism. Analogously, right $A$-homomorphisms are defined for right $A$-modules. For two-sided $A$ modules, a left and right $A$-homomorphism is called an $A$-homomorphism.

For left $\mathcal{T}$-modules $M$ and $N$ by $\operatorname{Hom}_{\mathcal{T}}(M, N)$, a family of all left $\mathcal{T}$-homomorphisms is defined from $M$ into $N$. A similar notation is used for a family of all $\mathcal{T}$-homomorphisms (or right $\mathcal{T}$-homomorphisms) of two-sided $\mathcal{T}$-modules (or right $\mathcal{T}$-modules correspondingly). If an algebra $A$ is specified, a homomorphism may be written for short, instead of an $A$-homomorphism.

Example 2. Let $\mathcal{T}$ be a commutative associative unital ring. Also, let $G$ be a metagroup and $A=\mathcal{T}[G]$ be a metagroup algebra, where $A$ is considered to be a $\mathcal{T}$-algebra. Put $K_{-1}=A, K_{0}=A \otimes_{\mathcal{T}} A$ and use induction $K_{n+1}=K_{n} \otimes_{\mathcal{T}}$ A for each natural number $n$. Each $K_{n}$ is supplied with a two-sided A-module structure: 
(1) $\forall p \in \mathcal{T}[\mathrm{C}(G)], p \cdot\left(x_{0}, \ldots, x_{n+1}\right)=\left(\left(p x_{0}\right), \ldots, x_{n+1}\right)$ and

$\left(x_{0}, \ldots,\left(x_{n+1} p\right)\right)=\left(x_{0}, \ldots, x_{n+1}\right) \cdot p$ and

$\forall j \in\{1, \ldots, n\}, p \cdot\left(x_{0}, \ldots, x_{n+1}\right)=\left(x_{0}, \ldots,\left(p x_{j}\right), \ldots, x_{n+1}\right)$ and

$\left(x_{0}, \ldots,\left(x_{j} p\right), \ldots, x_{n+1}\right)=\left(x_{0}, \ldots, x_{n+1}\right) \cdot p$,

where $0 \cdot\left(x_{1}, \ldots, x_{n}\right)=0$;

(2) $(x y) \cdot\left(x_{0}, \ldots, x_{n+1}\right)=\mathrm{t}_{3} \cdot\left(x \cdot\left(y \cdot\left(x_{0}, \ldots, x_{n+1}\right)\right)\right)$

with $\mathrm{t}_{3}=\mathrm{t}_{3}(x, y, b)$, (see also Formula (9) in Definition 1 above);

(3) $\mathrm{t}_{3} \cdot\left(\left(x_{0}, \ldots, x_{n+1}\right) \cdot(x y)\right)=\left(\left(x_{0}, \ldots, x_{n+1}\right) \cdot x\right) \cdot y$ with $\mathrm{t}_{3}=\mathrm{t}_{3}(b, x, y)$;

(4) $\left(x \cdot\left(x_{0}, \ldots, x_{n+1}\right)\right) \cdot y=\mathrm{t}_{3} \cdot\left(x \cdot\left(\left(x_{0}, \ldots, x_{n+1}\right) \cdot y\right)\right)$ with $\mathrm{t}_{3}=\mathrm{t}_{3}(x, b, y)$;

(5) $x \cdot\left(x_{0}, \ldots, x_{n+1}\right)=t_{n+3}\left(x, x_{0}, \ldots, x_{n+1} ; v_{0}(n+3) ; l(n+3)\right) \cdot\left(\left(x x_{0}\right), x_{1}, \ldots, x_{n+1}\right)$

where $\left\{x, x_{0}, \ldots, x_{n+1}\right\}_{v_{0}(n+3)}=x\left\{x_{0}, \ldots, x_{n+1}\right\}_{l(n+2)}$,

$\left\{x_{0}, \ldots, x_{n+1}\right\}_{l(n+2)}=\left\{x_{0}, \ldots, x_{n}\right\}_{l(n+1)} x_{n+1}$,

$\left\{x_{0}\right\}_{l(1)}=x_{0},\left\{x_{0} x_{1}\right\}_{l(2)}=x_{0} x_{1}$;

where $b=\left\{x_{0}, \ldots, x_{n+1}\right\}_{l(n+2)}$,

$t_{n}\left(x_{1}, \ldots, x_{n} ; u(n), w(n)\right):=t_{n}\left(x_{1}, \ldots, x_{n} ; u(n), w(n) \mid i d\right)$

using the shortened notation;

(6) $\left(x_{0}, \ldots, x_{n+1}\right) \cdot x=t_{n+3}\left(x_{0}, \ldots, x_{n+1}, x ; l(n+3), v_{n+2}(n+3)\right) \cdot\left(x_{0}, \ldots, x_{n},\left(x_{n+1} x\right)\right)$

for every $x, y, x_{0}, \ldots, x_{n+1}$ in $G$, where $\left(x_{0}, \ldots, x_{n+1}\right)$ denotes a basic element of $K_{n}$ over $\mathcal{T}$, corresponding to the left ordered tensor product

$$
\begin{aligned}
& \left(\ldots\left(\left(x_{0} \otimes x_{1}\right) \otimes x_{2}\right) \ldots \otimes x_{n}\right) \otimes x_{n+1}, \\
& \left\{x_{0}, \ldots, x_{n+1}, x\right\}_{v_{n+2}(n+3)}=\left\{x_{0}, \ldots, x_{n}, x_{n+1} x\right\}_{l(n+2)} .
\end{aligned}
$$

Proposition 1. For each metagroup algebra $A=\mathcal{T}[G]$ (see Definition 2), an acyclic left $A$-complex $\mathcal{K}$ exists.

Proof. Take two-sided $A$-modules $K_{n}$, as in example 2. We construct a boundary $\mathcal{T}$-linear operator $\partial_{n}: K_{n} \rightarrow K_{n-1}$ on $K_{n}$. For basic elements, it is given by the following formulas:

(1) $\partial_{n}\left(\left(x \cdot\left(x_{0}, x_{1}, \ldots, x_{n}, x_{n+1}\right)\right) \cdot y\right)=$

$\sum_{j=0}^{n}(-1)^{j} \cdot t_{n+4}\left(x, x_{0}, \ldots, x_{n+1}, y ; l(n+4), u_{j+1}(n+4)\right)$

$\cdot\left(\left(x \cdot\left(<x_{0}, x_{1}, \ldots, x_{n+1}>_{j+1, n+2}\right)\right) \cdot y\right)$, where

(2) $<x_{0}, \ldots, x_{n+1}>_{1, n+2}:=\left(\left(x_{0} x_{1}\right), x_{2}, \ldots, x_{n+1}\right)$,

(3) $<x_{0}, \ldots, x_{n+1}>_{2, n+2}:=\left(x_{0},\left(x_{1} x_{2}\right), x_{3}, \ldots, x_{n+1}\right), \ldots$,

(4) $<x_{0}, \ldots, x_{n+1}>_{n+1, n+2}:=\left(x_{0}, \ldots, x_{n-1},\left(x_{n} x_{n+1}\right)\right)$,

(5) $\partial_{0}\left(x \cdot\left(x_{0}, x_{1}\right)\right) \cdot y=\left(x \cdot\left(x_{0} x_{1}\right)\right) \cdot y$,

(6) $\left\{x_{0}, x_{1}, \ldots, x_{n+1}\right\}_{l(n+2)}:=\left(\ldots\left(\left(x_{0} x_{1}\right) x_{2}\right) \ldots\right) x_{n+1}$;

(7) $\left\{x, x_{0}, \ldots, x_{n+1}, y\right\}_{u_{1}(n+4)}:=\left(x\left\{\left(x_{0} x_{1}\right), x_{2}, \ldots, x_{n+1}\right\}_{l(n+1)}\right) y, \ldots$,

(8) $\left\{x, x_{0}, \ldots, x_{n+1}, y\right\}_{u_{n+1}(n+4)}:=\left(x\left\{x_{0}, x_{1}, \ldots,\left(x_{n} x_{n+1}\right)\right\}_{l(n+1)}\right) y$

for each $x, x_{0}, \ldots, x_{n+1}, y$ in $G$. On the other hand, from formulas (1) and (2) in Definition 1, it follows that $t_{n+4}\left(x, x_{0}, \ldots, x_{n+1}, y ; l(n+4), u_{j+1}(n+4)\right)=t_{n+2}\left(x_{0}, \ldots, x_{n+1} ; l(n+2), v_{j+1}(n+2)\right)$ for each $j=$ $0, \ldots, n$, where

(9) $\left\{x_{0}, \ldots, x_{n+1}\right\}_{v_{1}(n+2)}:=\left\{\left(x_{0} x_{1}\right), x_{2}, \ldots, x_{n+1}\right\}_{l(n+1)}, \ldots$,

(10) $\left\{x_{0}, \ldots, x_{n+1}\right\}_{v_{n+1}(n+2)}:=\left\{x_{0}, x_{1}, \ldots,\left(x_{n} x_{n+1}\right)\right\}_{l(n+1)}$

for every $x_{0}, \ldots, x_{n+1}$ in $G$. Therefore, $\partial_{n}$ is a left and right $A$-homomorphism of $(A, A)$-modules. In particular, $\partial_{1}\left(\left(x \cdot\left(x_{0}, x_{1}, x_{2}\right)\right) \cdot y\right)=\left(x \cdot\left(\left(x_{0} x_{1}\right), x_{2}\right)\right) \cdot y-\mathrm{t}_{3}\left(x_{0}, x_{1}, x_{2}\right) \cdot\left(x \cdot\left(x_{0},\left(x_{1} x_{2}\right)\right)\right) \cdot y$, 
$\partial_{2}\left(\left(x \cdot\left(x_{0}, x_{1}, x_{2}, x_{3}\right)\right) \cdot y\right)=\left(x \cdot\left(\left(x_{0} x_{1}\right), x_{2}, x_{3}\right)\right) \cdot y-t_{4}\left(x_{0}, \ldots, x_{3} ; l(4), v_{2}(4)\right) \cdot\left(\left(x \cdot\left(x_{0},\left(x_{1} x_{2}\right), x_{3}\right)\right) \cdot\right.$ $y)+t_{4}\left(x_{0}, \ldots, x_{3} ; l(4), v_{3}(4) ; i d\right)\left(\left(x \cdot\left(x_{0}, x_{1},\left(x_{2} x_{3}\right)\right)\right) \cdot y\right)$.

Define a $\mathcal{T}$-linear homomorphism $\mathbf{s}_{n}: K_{n} \rightarrow K_{n+1}$, which, for basic elements, has the form

(11) $\mathbf{s}_{n}\left(x_{0}, \ldots, x_{n+1}\right)=\left(1, x_{0}, \ldots, x_{n+1}\right)$ for every $x_{0}, \ldots, x_{n+1}$ in G. From Formulas (9) and (10) in Definition 1 and (1) in Lemma 1 the identities

(12) $t_{n}\left(x_{1}, \ldots, x_{n} ; q(n), u(n) \mid v\right) t_{n}\left(x_{1}, \ldots, x_{n} ; u(n), q(n) \mid v^{-1}\right)=1$

(13) $t_{n}\left(x_{1}, \ldots, x_{n} ; q(n), u(n)\right) t_{n}\left(x_{1}, \ldots, x_{n} ; u(n), w(n)\right)=t_{n}\left(x_{1}, \ldots, x_{n} ; q(n), w(n)\right)$ follow for every element $x_{1}, \ldots, x_{n}$ in metagroup $G$. Vectors $q(n), u(n)$, and $w(n)$ indicate the orders of their multiplication, $v \in S_{n}$ and $n \in \mathbf{N}$. The following identity is evident:

(14) $t_{n+1}\left(1, x_{1}, \ldots, x_{n} ; q(n+1), u(n+1) \mid v(n+1)\right)=t_{n}\left(x_{1}, \ldots, x_{n} ; q(n), u(n) \mid v(n)\right)$

for data $q(n), u(n)$ and $v(n)$ obtained from $q(n+1), u(n+1)$, and $v(n+1)$ correspondingly by taking the identity $1 b=b 1=b$ into account for each $b \in G$. Hence, $\mathbf{s}_{n}\left(\left(x_{0}, \ldots, x_{n+1}\right) \cdot y\right)=$ $\left(\mathbf{s}_{n}\left(x_{0}, \ldots, x_{n+1}\right)\right) \cdot y$ for every $x_{0}, \ldots, x_{n+1}, y$ in $G$.

Let $p_{n}: K_{n+1} \rightarrow K_{n}$ be a $\mathcal{T}$-linear mapping, such that

(15) $\quad p_{n}(a \otimes b)=a \cdot b$ and $p_{n}(b \otimes a)=b \cdot a$ for each $a \in K_{n}$ and $b \in A$. Therefore, from Formulas (13) and (14), we deduce that $p_{n} \mathbf{s}_{n}=i d$ is the identity on $K_{n}$. Consequently, $\mathbf{s}_{n}$ is a monomorphism.

Therefore, from Formulas (1), (11), (13), and (14) we infer that $\left(\partial_{n+1} \mathbf{s}_{n}+\mathbf{s}_{n-1} \partial_{n}\right)\left(x_{0}, \ldots, x_{n+1}\right)=$ $=\partial_{n+1}\left(1, x_{0}, \ldots, x_{n+1}\right)+\mathbf{s}_{n-1}\left(\sum_{j=0}^{n}(-1)^{j} t_{n+2}\left(x_{0}, \ldots, x_{n+1} ; l(n+2), v_{j+1}(n+2)\right) \cdot<x_{0}, \ldots, x_{n+1}>_{j+1, n+2}\right.$

)$)==\sum_{j=0}^{n+1}(-1)^{j} t_{n+3}\left(1, x_{0}, \ldots, x_{n+1} ; l(n+3), v_{j+1}(n+3)\right) \cdot<1, x_{0}, x_{1}, \ldots, x_{n+1}>_{j+1, n+3}+$ $+\sum_{j=0}^{n}(-1)^{j} t_{n+2}\left(x_{0}, \ldots, x_{n+1} ; l(n+2), v_{j+1}(n+2)\right) \cdot<1, x_{0}, \ldots, x_{n+1}>_{j+2, n+3}=\left(x_{0}, \ldots, x_{n+1}\right)$, for every $x_{0}, \ldots, x_{n+1}$ in $G$ (see also Definitions 2 and 3 and the notations above).

Thus, the homotopy conditions

(16) $\partial_{n+1} \mathbf{s}_{n}+\mathbf{s}_{n-1} \partial_{n}=1$ for each $n \geq 0$

are fulfilled, where 1 denotes the identity operator on $K_{n}$. Therefore, the recurrence relation

(17) $\partial_{n} \partial_{n+1} \mathbf{s}_{n}=\mathbf{s}_{n-2} \partial_{n-1} \partial_{n}$

is accomplished, since

$\partial_{n} \partial_{n+1} \mathbf{s}_{n}=\partial_{n}\left(1-\mathbf{s}_{n-1} \partial_{n}\right)=\partial_{n}-\left(\partial_{n} \mathbf{s}_{n-1}\right) \partial_{n}=\partial_{n}-\left(1-\mathbf{s}_{n-2} \partial_{n-1}\right) \partial_{n}$.

On the other hand, from Formula (11), it follows that $K_{n+1}$, as the left $A$-module, is generated by $\mathbf{s}_{n} K_{n}$. Then, proceeding by induction in $n$ with the help of (17), we deduce that $\partial_{n} \partial_{n+1}=0$ for each $n \geq 0$, since $\partial_{0} \partial_{1}=0$ according to Formulas (1) and (5).

An opposite algebra $A^{o p}$ exists. The latter, as an $\mathcal{T}$-linear space, is the same, but has the multiplication $x \circ y=y x$ for each $x, y \in A^{o p}$. Let $A^{e}:=A \otimes_{\mathcal{T}} A^{o p}$ denote the enveloping algebra of $A$. Apparently, $K_{0}=A \otimes_{\mathcal{T}} A$ coincides with $A \otimes_{\mathcal{T}} A^{o p}$ as a left and right $A$-module. Hence, the mapping $\partial_{0}: K_{0} \rightarrow K_{-1}$ provides the augmentation $\epsilon: A^{e} \rightarrow A$.

Thus, identity (16) means that the left complex $\mathcal{K} 0 \leftarrow A \overleftarrow{\jmath_{0}} K_{0} \overleftarrow{\jmath_{1}} K_{1} \overleftarrow{\jmath_{2}} K_{2} \leftarrow . . \overleftarrow{\jmath_{n}} K_{n} \overleftarrow{\jmath_{n+1}} K_{n+1} \leftarrow \ldots$ is acyclic.

Example 3. For the Cayley-Dickson algebra $A_{n}$ over a field $F$ of characteristics not equal to two, let $G=G_{n}$, as the (multiplicative) metagroup, consist of all elements $b i_{k}$ with $b \in \mathbf{\Psi}, k=0,1,2, \ldots$, where $i_{0}, i_{1}, i_{2}, \ldots$ are generators of the Cayley-Dickson algebra $A_{n}, 2 \leq n \leq \infty$. Then, $M=A_{n}^{j}$ is the module over $\mathbf{Z}[G]$, where $j \in \mathbf{N}$.

Example 4. For a topological space $U$, it is possible to consider the module $M=C\left(U, A_{n}^{j}\right)$ of all continuous mappings from $U$ into $A_{n}^{j}, j \in \mathbf{N}, A_{n}^{j}$, which is supplied with the box product topology.

Example 5. If $(U, \mathcal{B}, \mu)$ is a measure space, where $\mu: \mathcal{B} \rightarrow[0, \infty)$ is a $\sigma$-additive measure on a $\sigma$-algebra $\mathcal{B}$ of a set $U$, for $\mathbf{F}=\mathbf{R}$ and $f_{k}=1$ for each $k$, it is possible to consider the space $L_{p}\left((U, \mathcal{B}, \mu), A_{n}^{j}\right)$ of all $L_{p}$ 
mappings from $U$ into $A_{n}^{j}$, where $A_{n}$ is taken relative to its norm induced by the scalar product $\operatorname{Re}(\bar{y} z)=(y, z)$, $j \in \mathbf{N}, 1 \leq p \leq \infty$.

Example 6. For an additive group $H$, one can consider the trivial action of $A$ on $H$. Therefore, the direct product $M \otimes H$ becomes an $A$-module for an $A$-module $M$. In particular, $H$ may be a ring.

Example 7. If there is another ring $\mathcal{S}$ and a homomorphism $\phi: \mathcal{S} \rightarrow \mathcal{T}$, then each left (or right) $\mathcal{T}$-module $M$ can be considered as a left (or right, correspondingly) $\mathcal{S}$-module by the rule bm=( $b b) m$ (or $m b=m(\phi b)$ correspondingly) for each $b \in \mathcal{S}$ and $m \in M$.

Vice versa, if $M$ is a right (or left) $\mathcal{S}$-module, then the right (or left, correspondingly) module exists $M_{(\phi)}=M \otimes_{\mathcal{S}} \mathcal{T}$ (or ${ }_{(\phi)} M=\mathcal{T} \otimes_{\mathcal{S}} M$, correspondingly), which is called the right (or left correspondingly) covariant $\phi$-extension of $M$. Similarly, the contravariant right and left extensions $M^{(\phi)}=\operatorname{Hom}_{\mathcal{S}}(\mathcal{T}, M)$ or ${ }^{(\phi)} M$ are defined for right or left $\mathcal{S}$-modules $M$, respectively.

This also can be applied to a metagroup algebra $A=\mathcal{S}[G]$ over a commutative associative unital ring $\mathcal{S}$ as in Example 1. Then, by changing a ring, we get right $A_{(\phi)}$ or $A^{(\phi)}$ and left ${ }_{(\phi)} A$ or ${ }^{(\phi)} A$ algebras over $\mathcal{T}$. Then, imposing the relation $t a=a$ for each $a \in A$ and $t \in T$ provides a metagroup algebra over $\mathcal{T}$, which also has a two-sided $\mathcal{T}$-module structure. It will be denoted by ${ }_{(\phi)} A_{(\phi)}$ or ${ }^{(\phi)} A^{(\phi)}$, respectively. Particularly, this is applicable to cases when $\mathbf{Z}[\mathbf{\Psi}] \subset \mathcal{S}$ or $\phi$ is an embedding.

Notation 1. Let $A=\mathcal{T}[G]$ be a metagroup algebra (see Definition 2). Put $L_{0}=\mathcal{T}, L_{1}=A$ and by induction, $L_{n+1}=L_{n} \otimes_{\mathcal{T}}$ A for each natural number $n$.

If $N$ is a two-sided $A$-module, it can also be considered as a left $A^{e}$-module by the rule $\left(x \otimes y^{*}\right) b:=$ $(x \otimes b) \otimes y$ for each $x \in A, y^{*} \in A^{o p}$, and $b \in N, A^{e}=A \otimes \mathcal{T} A^{o p}$ is an enveloping algebra, where $A^{o p}$ denotes the opposite algebra of $A$, where $y^{*}$ in $A^{\text {op }}$ corresponds to $y$ in element $A$.

Theorem 1. If $\mathcal{K}$ is an acyclic left $A$-complex for a metagroup algebra $A=\mathcal{T}[G]$, as in Proposition 1 , and $M$ is a two-sided A-module satisfying Conditions $(1-3)$ in Definition 3, then a co-chain complex $\operatorname{Hom}(\mathcal{L}, M)$ exists:

(1) $\quad 0 \rightarrow \operatorname{Hom}_{\mathcal{T}}\left(L_{0}, M\right) \underset{\epsilon^{*}}{\rightarrow} \operatorname{Hom}_{\mathcal{T}}\left(L_{1}, M\right) \underset{\delta^{1}}{\rightarrow} \operatorname{Hom}_{\mathcal{T}}\left(L_{2}, M\right) \underset{\delta^{2}}{\rightarrow} \operatorname{Hom}_{\mathcal{T}}\left(L_{3}, M\right) \underset{\delta^{3}}{H} \operatorname{Hom}_{\mathcal{T}}\left(L_{4}, M\right) \underset{\delta^{4}}{\cdots}$

such that $f \in \operatorname{Hom}_{\mathcal{T}}\left(L_{1}, M\right)$ is a co-cycle, if and only if $f$ is a $\mathcal{T}$-linear derivation from $A$ into $M$.

Proof. Notation 1 and Example 2 permit each basic element $\left(x_{0}, \ldots, x_{n+1}\right)$ of $K_{n}$ over $\mathcal{T}$ to be written as

(1) $\left(x_{0}, \ldots, x_{n+1}\right)=t_{n+2}\left(x_{0}, \ldots, x_{n+1} ; l(n+2), w(n+2)\right) \cdot\left(\left(x_{0} \otimes\left(x_{1}, \ldots, x_{n}\right)\right) \otimes x_{n+1}\right)$ and

(2) $\left(x_{0}, \ldots, x_{n+1}\right)=t_{n+2}\left(x_{0}, \ldots, x_{n+1} ; l(n+2), w(n+2)\right) \cdot\left(z \otimes\left(x_{1}, \ldots, x_{n}\right)\right)$, where $\left(x_{1}, \ldots, x_{n}\right)$ is a basic element in $L_{n}$ for every $x_{0}, \ldots, x_{n+1}$ in $G,\left\{x_{0}, \ldots, x_{n+1}\right\}_{w(n+2)}=\left(x_{0}\left\{x_{1}, \ldots, x_{n}\right\}_{l(n)}\right) x_{n+1}, z \in A^{e}$, $z=x_{0} \otimes x_{n+1}^{*}$.

Each homomorphism $f \in \operatorname{Hom}_{\mathcal{T}}\left(L_{n}, M\right)$ is characterized by its values on elements $\left(x_{1}, \ldots, x_{n}\right)$, where $x_{1}, . ., x_{n}$ belong to a metagroup $G$. Consider $f$ as a $\mathcal{T}$-linear function from $A^{n}$ into $M$. Since $M$ satisfies Conditions $(1-3)$ in Definition 3 , then $f$ has the decomposition

(2) $f\left(x_{1}, \ldots, x_{n}\right)=\sum_{g \in G} f_{g}\left(x_{1}, \ldots, x_{n}\right)$,

where $f_{g}: G^{n} \rightarrow M_{g}$ for every $g$ and $x_{1}, \ldots, x_{n}$ in $G$.

Therefore, the restrictions follow from Conditions $(1-3)$ in Definition 3 , which take into account the nonassociativity of $G$ :

(3) $(x y) \cdot f_{g}\left(x_{1}, \ldots, x_{n}\right)=\mathrm{t}_{3}(x, y, g) \cdot\left(x \cdot\left(y \cdot f_{g}\left(x_{1}, \ldots, x_{n}\right)\right)\right)$,

(4) $\mathrm{t}_{3}(g, x, y) \cdot\left(f_{g}\left(x_{1}, \ldots, x_{n}\right) \cdot(x y)\right)=\left(f_{g}\left(x_{1}, \ldots, x_{n}\right) \cdot x\right) \cdot y$, 
(5) $\left(x \cdot f_{g}\left(x_{1}, \ldots, x_{n}\right)\right) \cdot y=\mathrm{t}_{3}(x, g, y) \cdot\left(x \cdot\left(f_{g}\left(x_{1}, \ldots, x_{n}\right) \cdot y\right)\right)$

for every $g$ and $x, y, x_{1}, \ldots, x_{n}$ in $G$, where coefficients $t_{3}$ are prescribed by Formula (9) in Definition 1. Also,

(6) $x \cdot f_{g}\left(x_{1}, \ldots, x_{n}\right):=x \cdot\left(f_{g}\left(x_{1}, \ldots, x_{n}\right)\right)$ and

(7) $f_{g}\left(x_{1}, \ldots, x_{n}\right) \cdot y:=\left(f_{g}\left(x_{1}, \ldots, x_{n}\right)\right) \cdot y$.

For $n=0$ and $g=e$, naturally, the identities are fulfilled:

(8) $\quad(x y) \cdot f_{e}()=x \cdot\left(y \cdot f_{e}()\right),\left(f_{e}() \cdot x\right) \cdot y=f_{e}() \cdot(x y)$ and $\left(x \cdot f_{e}()\right) \cdot y=x \cdot\left(f_{e}() \cdot y\right)$.

A co-boundary operator exists that takes into account the nonassociativity of the (multiplicative) metagroup G:

(9) $\left(\delta^{n} f\right)\left(x_{1}, \ldots, x_{n+1}\right)=\sum_{j=0}^{n+1}(-1)^{j} t_{n+1}\left(x_{1}, \ldots, x_{n+1} ; l(n+1), u_{j+1}(n+1)\right) \cdot\left[f, x_{1}, x_{2}, \ldots, x_{n+1}\right]_{j+1, n+1}$, where

(10) $\left[f, x_{1}, \ldots, x_{n+1}\right]_{1, n+1}:=x_{1} \cdot f\left(x_{2}, \ldots, x_{n+1}\right),\left\{x_{1}, \ldots, x_{n+1}\right\}_{u_{1}(n+1)}=x_{1}\left\{x_{2}, \ldots, x_{n+1}\right\}_{l(n)}$;

(11) $\left[f, x_{1}, \ldots, x_{n+1}\right]_{2, n+1}:=f\left(\left(x_{1} x_{2}\right), \ldots, x_{n+1}\right),\left\{x_{1}, \ldots, x_{n+1}\right\}_{u_{2}(n+1)}=\left\{\left(x_{1} x_{2}\right), \ldots, x_{n+1}\right\}_{l(n)} ; \ldots ;$

(12) $\left[f, x_{1}, \ldots, x_{n+1}\right]_{n+1, n+1}:=f\left(x_{1}, x_{2}, \ldots,\left(x_{n} x_{n+1}\right)\right) ;\left\{x_{1}, \ldots, x_{n+1}\right\}_{u_{n+1}(n+1)}=\left\{x_{1}, \ldots,\left(x_{n} x_{n+1}\right)\right\}_{l(n)}$;

(13) $\left[f, x_{1}, \ldots, x_{n+1}\right]_{n+2, n+1}:=f\left(x_{1}, x_{2}, \ldots, x_{n}\right) \cdot x_{n+1},\left\{x_{1}, \ldots, x_{n+1}\right\}_{u_{n+2}(n+1)}=\left\{x_{1}, \ldots, x_{n+1}\right\}_{l(n+1)}=$ $\left(\ldots\left(\left(x_{1} x_{2}\right) x_{3}\right) \ldots x_{n}\right) x_{n+1}$; with $u_{0}(n+1)=l(n+1)$.

From $G^{n+1}$ onto $K_{n+1}$, the homomorphism $\left(\delta^{n} f\right)$ is extended by the $\mathcal{T}$-linearity. On the other hand, Condition (1) in Definition 3 implies that

(14) For each $b \in G, h_{1, b}$ exists, so that $h_{1, b}: K_{n+1} \rightarrow M_{1}$ and $f_{b}=h_{1, b} L_{b}$, where $L_{b}$ is the left multiplication operator on $b$ :

(15) $\left(h_{1, b} L_{b}\right)\left(x_{1}, \ldots, x_{n}\right)=b \cdot\left(h_{1, b}\left(x_{1}, \ldots, x_{n}\right)\right)$ for every $x_{1}, \ldots, x_{n}$ in $G$. Moreover, $z g=0$ (or $\left.g z=0\right)$ in $\mathbf{Z}[G]$ for $g \in G$ and $z \in \mathbf{Z}[G]$, if and only if $z=0$, since $G$ is a metagroup.

By virtue of Proposition 1, these formulas imply that $\delta^{n+1} \circ \delta^{n}=0$ for each $n$, since $\left(\delta^{n+1} \circ\right.$ $\left.\delta^{n} f\right)\left(x_{1}, \ldots, x_{n+2}\right)=f\left(\partial_{n-1} \circ \partial_{n}\left(x_{1}, \ldots, x_{n+2}\right)\right)$ for every $x_{1}, \ldots, x_{n+2}$ in $G$. Thus, the complex given by formula (1) is exact.

Particularly, $f \in \operatorname{Hom}_{\mathcal{T}}\left(L_{0}, M\right)$ is a co-cycle if and only if

$\left(\delta^{0} f\right)(x)=x f()-f() x=0$ for each $x \in G$.

We mention that $\operatorname{Hom}_{\mathcal{T}}\left(L_{0}, M\right)$ is isomorphic with $M$.

The one-dimensional co-chain $f \in \operatorname{Hom}_{\mathcal{T}}\left(L_{1}, M\right)$ is determined by the mapping $f: G \rightarrow M$. Taking Formula (9) into account, we infer that it is a co-cycle if and only if

$$
\begin{aligned}
& t_{2}\left(x, y ; l(2), u_{1}(2)\right) \cdot x \cdot f(y)-t_{2}\left(x, y ; l(2), u_{2}(2)\right) \cdot f(x y)+t_{2}\left(x, y ; l(2), u_{3}(2)\right) \cdot f(x) \cdot y=x \cdot f(y)- \\
& f(x y)+f(x) \cdot y=0
\end{aligned}
$$

for each $x$ and $y$ in $G$. That is, $f$ is a derivation from the metagroup $G$ into the $G$-module $M$. There is the embedding $\mathcal{T} \hookrightarrow A$ of $\mathcal{T}$ into $A$ as $\mathcal{T} e$, since $e=1 \in G$. Thus, $f$ has a $\mathcal{T}$-linear extension to a $\mathcal{T}$-linear derivation from $A$ into $M$ by the following formula:

$$
f(x y)=x \cdot f(y)+f(x) \cdot y .
$$

Remark 1. Suppose that the conditions of Theorem 1 are fulfilled. A two-dimensional co-chain is a 2-co-cycle, if and only if $\left(\delta^{2} f\right)\left(x_{1}, x_{2}, x_{3}\right)=\sum_{j=0}^{3}(-1)^{j} t_{3}\left(x_{1}, x_{2}, x_{3} ; l(3), u_{j+1}(3)\right)$. $\left[f, x_{1}, x_{2}, x_{3}\right]_{j+1,3}=t_{3}\left(x_{1}, x_{2}, x_{3} ; l(3), u_{1}(3)\right) \cdot x_{1} \cdot f\left(x_{2}, x_{3}\right)-t_{3}\left(x_{1}, x_{2}, x_{3} ; l(3), u_{2}(3)\right) \cdot f\left(\left(x_{1} x_{2}\right), x_{3}\right)+$ $t_{3}\left(x_{1}, x_{2}, x_{3} ; l(3), u_{3}(3)\right) \cdot f\left(x_{1},\left(x_{2} x_{3}\right)\right)-t_{3}\left(x_{1}, x_{2}, x_{3} ; l(3), u_{4}(3)\right) \cdot f\left(x_{1}, x_{2}\right) \cdot x_{3}=0$.

That is,

(1) $\mathrm{t}_{3}\left(x_{1}, x_{2}, x_{3}\right) \cdot x_{1} \cdot f\left(x_{2}, x_{3}\right)+\mathrm{t}_{3}\left(x_{1}, x_{2}, x_{3}\right) \cdot f\left(x_{1},\left(x_{2} x_{3}\right)\right)=f\left(\left(x_{1} x_{2}\right), x_{3}\right)+f\left(x_{1}, x_{2}\right) \cdot x_{3}$ 
for each $x_{1}, x_{2}$ and $x_{3}$ in $G$.

Usually, $Z^{n}(A, M)$ denotes the set of all $n$-co-cycles, and the notation $B^{n}(A, M)$ is used for the set of $n$-co-boundaries in $H_{\mathcal{T}}\left(L_{n}, M\right)$. Since, as the additive group, $M$ is commutative, then there are defined groups of cohomologies $H^{n}(A, M)=Z^{n}(A, M) / B^{n}(A, M)$ as the quotient (additive) groups.

For $n=0$, the co-boundaries are set as zero, and hence, $H^{0}(A, M) \cong M^{A}$. In the case $n=1$, a mapping $f: A \rightarrow M$ is a co-boundary if the element $m=h() \in M$ exists, for which $f(x)=x m-m x$ for each $x \in A$. Such a derivation $f$ is called an inner derivation of $A$ defined by an element $m \in M$. The set of all inner derivations is denoted by $\operatorname{Inn} \mathcal{T}(A, M)$.

From the cohomological point of view, the additive group $H^{1}(A, M)$ is interpreted as the group of all outer derivations $H^{1}(A, M) \cong \operatorname{Out}_{\mathcal{T}}(A, M) \cong \operatorname{Der}_{\mathcal{T}}(A, M) / \operatorname{Inn}_{\mathcal{T}}(A, M)$, where $Z^{1}(A, M)=$ $\operatorname{Der}_{\mathcal{T}}(A, M) ; \quad \operatorname{Inn}_{\mathcal{T}}(A, M)=B^{1}(A, M)$, where the family of all derivations $(\mathcal{T}$-homogeneous derivations) from $X$ into a two-sided module $M$ over $\mathcal{T}$ is denoted by $\operatorname{Der}(X, M)\left(\right.$ or $\operatorname{Der}_{\mathcal{T}}(X, M)$ respectively).

A two-co-chain $f: G \times G \rightarrow M$ is a two-co-boundary, if a one-co-chain $h: G \rightarrow M$ exists such that for each $x$ and $y$ in $G$, the following identity is fulfilled:

$$
\begin{aligned}
& f(x, y)=(\delta h)(x, y)=\sum_{j=0}^{2}(-1)^{j} t_{2}\left(x, y ; l(2), u_{j+1}(2)\right) \cdot\left[h, x_{1}, x_{2}\right]_{j+1,2}, \\
& =x \cdot h(y)-h(x y)+h(x) \cdot y .
\end{aligned}
$$

Let $A=\mathcal{T}[G]$ be a metagroup algebra over a commutative associative unital ring $\mathcal{T}$ (also see Definitions 1-3).

Let $M, N$, and $P$ be left $A$-modules, and a short exact sequence exists:

(3) $0 \rightarrow M_{\vec{\xi}} P_{\vec{\eta}} N \rightarrow 0$,

where $\xi$ is an embedding, such that $\xi$ and $\eta$ are left $A$-homomorphisms. Then, $P$ is called an enlargement of a left $A$-module $M$ with the help of a left $A$-module $N$. If there is another enlargement of $M$ with the help of $N$,

$$
0 \rightarrow M_{\xi^{\prime}} P^{\prime} \underset{\eta^{\prime}}{\rightarrow} N \rightarrow 0
$$

such that an isomorphism $\pi: P \rightarrow P^{\prime}$ exists for which $\pi \xi=\xi^{\prime} 1_{M}$ and $1_{N} \eta=\eta^{\prime} \pi$, then enlargements (3) and (4) are called equivalent, where $1_{M}: M \rightarrow M$ notates the identity mapping, $1_{M}(m)=m$ for each $m \in M$.

It is said that an enlargement clefts, if and only if a left $A$-homomorphism $\omega: N \rightarrow P$ exists, fulfilling the restriction $\eta \omega=1_{N}$.

In the particular case when $P=M \oplus N, \xi$ is also an identifying mapping with the first direct summand, and $\eta$ is a projection on the second direct summand, an enlargement is called trivial.

Theorem 2. Let $A$ be a nonassociative metagroup algebra and let $M$ and $N$ be left $A$-modules, where $A=\mathcal{T}[G]$, $G$ is a metagroup (see Definitions 2 and 3). Then, the family $T=\operatorname{Hom}_{\mathcal{T}}(N, M)$ can be supplied with a two-sided A-module structure, such that $H^{1}(A, T)$ is the set of classes of modules $M$ with the quotient module $N$.

Proof. The family $T=\operatorname{Hom}_{\mathcal{T}}(N, M)$ evidently has the structure of a left module over a ring $\mathcal{T}$ (see Definition 3), and it can be supplied with a two-sided $A$-module structure:

(1) $\forall r \in T$ and $\forall n \in N$ and $\forall a \in A$ :

$(a \cdot r)(n)=a \cdot(r(n))$ and $(r \cdot a)(n)=r(a \cdot n)$.

By virtue of Theorem 1 , each element $f \in Z^{1}(A, T)$ induces a (generalized) derivation by Formula (18) in Theorem 1. Each zero-dimensional co-chain $m \in M$ provides an inner derivation $\delta^{0} m(a)=a m-m a$ due to formula (16) in Theorem 1. Then, one co-cycle $f$ induces an enlargement by Formula (3) in Remark 1 with $P=M \oplus N$ being the direct sum of left $A$-modules in which $N$ is a submodule and with the left action of $A$ on $N: a \circ n=a \cdot n+f(a) \cdot n$ for each $n \in N$ and $a \in A$. 
Suppose that a class of an one co-cycle $f$ is zero, an element $u \in T$ exists so that $f=\delta^{1} u$. Then, elements of the form $m+u(m)$ form its submodule $M^{\prime}$, which is isomorphic with $M$. Moreover, $P=N \oplus M^{\prime}$ is the direct sum of $A$-modules. Thus, an enlargement is trivial.

Vice versa, suppose that an enlargement given by formula (3) in Remark 1 exists. That is, a left $A$-homomorphism $\gamma: N \rightarrow P$ satisfying the restriction $\eta \gamma=1_{N}$ exists, where $1_{N}$ notates the identity mapping on $N$. It induces $f \in Z^{1}(A, T)$, such that $f(a) n=\gamma(a \cdot n)-(a \cdot \gamma)(n)$ for all $n \in N$ and for each element $a$ of the algebra $A$.

Suppose that there is another enlargement which clefts, that is, a left $\mathcal{T}$-homomorphism $\omega: N \rightarrow P$ exists, fulfilling the restriction $\eta \omega=1_{N}$. We put $u(n)=\omega(n)-\gamma(n)$ for each $n \in N$; hence, $u \in T$. Then, $f_{1}=\delta^{1} u$ is a co-cycle of zero class.

Theorem 3. Suppose that $A$ is a nonassociative metagroup algebra over a commutative associative unital ring $\mathcal{T}$, a left $A$-module $N$ and a two-sided $A$-module $M$ are given. Then, $H^{2}(A, T)$ is the set of classes of enlargements of $A$ with a kernel $M$ such that $M^{2}=\{0\}$ and with the quotient algebra A. Moreover, an action of $A$ on $M$ in this enlargement coincides with the structure of a two-sided A-module on $M$.

Proof. If $P$ is an enlargement with a kernel $M$ such that $M^{2}=0$ and a quotient module $A=P / M$ and $a=p+M$ with $p \in P$, then $a \cdot m=p \cdot m$ and $m \cdot a=m \cdot p$ supply $M$ with the two-sided $A$-module structure. Take a $\mathcal{T}$-linear mapping $\gamma: A \rightarrow P$ inverse from the left to a natural epimorphism and put $f(a, b)=\gamma(a b)-\gamma(a) \gamma(b)$ for each $a$ and $b$ in $A$. Then, we infer that $\gamma(a(b c))=f(a, b c)+\gamma(a) \gamma(b c)=f(a, b c)+\gamma(a)(f(b, c)+\gamma(b) \gamma(c))$ and $\gamma((a b) c)=f(a b, c)+$ $\gamma(a b) \gamma(c)=f(a b, c)+(f(a, b)+\gamma(a) \gamma(b)) \gamma(c)$, consequently, $0=\mathrm{t}_{3}(a, b, c) \gamma(a(b c))-\gamma((a b) c)=$ $\mathrm{t}_{3}(a, b, c) f(a, b c)+\mathrm{t}_{3}(a, b, c) \gamma(a)(f(b, c)+\gamma(b) \gamma(c))-f(a b, c)-(f(a, b)+\gamma(a) \gamma(b)) \gamma(c)$.

Taking into account that $\gamma(a) m=a \cdot m$ and $m \gamma(a)=m \cdot a$ for each $m \in M$ and $a \in A$, we deduce using Formula (1) in Remark 1 that $0=\mathrm{t}_{3}(a, b, c) \cdot a \cdot f(b, c)-f(a b, c)+\mathrm{t}_{3}(a, b, c) \cdot f(a, b c)-f(a, b)$. $c=\left(\delta^{2} f\right)(a, b, c)$.

Thus, $f \in B^{2}(A, M)$ and hence, $f=\left(\delta^{1} h\right)$ with $h \in C^{1}(A, M):=\operatorname{Hom}_{\mathcal{T}}(A, M)$.

It remains to prove that the set $S$ of all elements $\gamma(a)+h(a)$ forms a subalgebra isomorphic with $A$ in $P$. From the construction of $S$, it follows that $S$ is a a two-sided $\mathcal{T}$-module. We verify that it is closed relative to the multiplication for all $a$ and $b$ in $A$ :

$(\gamma(a)+h(a))(\gamma(b)+h(b))=\gamma(a) \gamma(b)+\gamma(a) h(b)+h(a) \gamma(b)=\gamma(a b)-f(a, b)+a h(b)+$ $h(a) b=\gamma(a b)+h(a b)+a h(b)-h(a b)+h(a) b-f(a, b)=\gamma(a b)+h(a b)+\left(\delta^{1} h\right)(a, b)-f(a, b)=$ $\gamma(a b)+h(a b)$.

If $A, M$, and $f$ are given, then an enlargement $P$ can be constructed as the direct sum $P=M \oplus A$ of two-sided $\mathcal{T}$-modules and with the multiplication rule $\left(m_{1}+b_{1}\right)\left(m_{2}+b_{2}\right)=m_{1} b_{2}+m_{2} b_{1}+f\left(b_{1}, b_{2}\right)+$ $b_{1} b_{2}$ for every $m_{1}$ and $m_{2}$ in $M$ and $b_{1}$ and $b_{2}$ in $A$. It rests to verify that this multiplication rule is homogeneous over $\mathcal{T}$ and right and left distributive. At first, we evidently get $\left(m_{1}+b_{1}\right)\left(s\left(m_{2}+\right.\right.$ $\left.\left.b_{2}\right)\right)=\left(s\left(m_{1}+b_{1}\right)\right)\left(m_{2}+b_{2}\right)=s\left(\left(m_{1}+b_{1}\right)\left(m_{2}+b_{2}\right)\right)=s m_{1} b_{2}+s m_{2} b_{1}+s f\left(b_{1}, b_{2}\right)+s b_{1} b_{2}$ and $(s p)\left(m_{1}+b_{1}\right)=s\left(p\left(m_{1}+b_{1}\right)\right)$ for all $s, p \in \mathcal{T}$ and $m_{1}$ and $m_{2}$ in $M$ and $b_{1}$ and $b_{2}$ in $A$, since $\mathcal{T} \subset$ $\mathcal{C}(A)$ and $f(s, p)=0$. Moreover, we infer that $\left(m_{1}+b_{1}\right)\left(\left(m_{2}+b_{2}\right)+\left(m_{3}+b_{3}\right)\right)=\left(m_{1}+b_{1}\right)\left(\left(m_{2}+\right.\right.$ $\left.\left.m_{3}\right)+\left(b_{2}+b_{3}\right)\right)=m_{1}\left(b_{2}+b_{3}\right)+\left(m_{2}+m_{3}\right) b_{1}+f\left(b_{1}, b_{2}+b_{3}\right)+b_{1}\left(b_{2}+b_{3}\right)=m_{1} b_{2}+m_{1} b_{3}+m_{2} b_{1}+$ $m_{3} b_{1}+f\left(b_{1}, b_{2}\right)+f\left(b_{1}, b_{3}\right)+b_{1} b_{2}+b_{1} b_{3}=\left(m_{1}+b_{1}\right)\left(m_{2}+b_{2}\right)+\left(m_{1}+b_{1}\right)\left(m_{3}+b_{3}\right)$, and analogously, $\left(\left(m_{1}+b_{1}\right)+\left(m_{2}+b_{2}\right)\right)\left(m_{3}+b_{3}\right)=\left(m_{1}+b_{1}\right)\left(m_{3}+b_{3}\right)+\left(m_{2}+b_{2}\right)\left(m_{3}+b_{3}\right)$ for all $m_{1}, m_{2}$ and $m_{3}$ in $M$ and $b_{1}, b_{2}$ and $b_{3}$ in $A$.

Definition 4. Let $M$ and $P$ and $N$ be two-sided $A$-modules, where $A$ is a nonassociative metagroup algebra over a commutative associative unital ring $\mathcal{T}$. An A-homomorphism (isomorphism) $f: M \rightarrow P$ is called a right (operator) A-homomorphism (isomorphism) if it is such for $M$ and $N$ as right A-modules, that is, $f(x+y)=$ $f(x)+f(y)$ and $f(x a)=f(x)$ a for each $x$ and $y$ in $M$ and $a \in A$ (see also Definition 3). If an algebra $A$ is specified, a homomorphism (isomorphism) may be written for short instead of an A-homomorphism (an A-isomorphism, respectively). 
An enlargement $(P, \eta)$ of $M$ by $N$ is called right inessential if a right isomorphism $\gamma: N \rightarrow P$ exists, satisfying the restriction $\left.\eta \gamma\right|_{N}=\left.1\right|_{N}$.

Theorem 4. Suppose that $M$ is a two-sided A-module, where $A$ is a nonassociative metagroup algebra over a commutative associative unital ring $\mathcal{T}$. Then, for each $n \geq 0$, a two-sided $A$-module $P_{n}$ exists such that $H^{n+1}(A, M)$ is isomorphic with the additive group of equivalence classes of right inessential enlargements of $M$ by $P_{n}$.

Proof. Consider two right inessential enlargements $\left(E_{1}, \eta_{1}\right)$ and $\left(E_{2}, \eta_{2}\right)$ of $M$ by $N$, where $\xi_{1}$ and $\xi_{2}$ are embeddings of $M$ into $E_{1}$ and $E_{2}$ correspondingly. Take a submodule $Q$ of $E_{1} \oplus E_{2}$ consisting of all elements $\left(x_{1}, x_{2}\right)$ satisfying the condition $\eta_{1}\left(x_{1}\right)=\eta_{2}\left(x_{2}\right)$. Then, a quotient module $Q / T$ exists, where $T=\{(\xi m,-\xi m): m \in M\}$. Therefore, $\left(\xi_{1} M \oplus \xi_{2} M\right) / T$ is isomorphic with $M$, and homomorphisms $\eta_{1}$ and $\eta_{2}$ induce a homomorphism $\eta$ of $Q / T$ onto $N$. Hence, the submodule $\operatorname{ker}(\eta)$ is isomorphic with $M$. Then, an addition of enlargements is prescribed by the formula $\left(E_{1}, \eta_{1}\right)+\left(E_{2}, \eta_{2}\right):=(Q / T, \eta)$. Evidently, sums of equivalent enlargements are equivalent.

For an enlargement $(E, \eta)$ of $M$ by $N$, one takes the direct sum of modules $E \oplus M$ and puts $T_{b}$ to be its submodule consisting of all elements $(\xi m,-b \xi m)$ with $m \in M$, where $\xi$ is an embedding of $M$ into $E, b \in \mathcal{T}$. Therefore, a homomorphism $\eta$ induces a homomorphism ${ }_{b} \eta$ of $(E \oplus M) / T_{b}$ onto $N$, since the mapping $(\xi m, m) \mapsto b \xi m+m$ is a homomorphism of $(\xi M) \oplus M$ onto $M$. Also, the ring $\mathcal{T}$ is commutative and associative. This induces an enlargement of $M$ by $N$, denoted by $\left({ }_{b} E,{ }_{b} \eta\right)$ and hence, an operation of scalar multiplication of an enlargement $(E, \eta)$ on $b \in \mathcal{T}$. From this construction, it follows that equivalent enlargements have equivalent scalar multipliers on $b \in \mathcal{T}$.

Let $P_{n}$ be a $\mathcal{T}$-linear span of all elements $\left(x_{1}, \ldots, x_{n+1}\right)$ with $x_{1}, \ldots, x_{n+1}$ in $G$ such that $\left(\left(b x_{1}\right), x_{2}, \ldots, x_{n+1}\right)=\left(x_{1}, \ldots,\left(b x_{n+1}\right)\right)$ for each $b \in \mathcal{T}$. Next, we put

(1) $\left(x_{1}, \ldots, x_{n+1}\right) \cdot y:=t_{n+2}\left(x_{1}, \ldots, x_{n+1}, y ; l(n+2), u_{n+2}(n+2)\right) \cdot\left(x_{1}, \ldots, x_{n},\left(x_{n+1} y\right)\right)$ and

(2) $y \cdot\left(x_{1}, \ldots, x_{n+1}\right)=\sum_{j=1}^{n+1}(-1)^{j+1}$.

$$
t_{n+2}\left(y, x_{1}, \ldots, x_{n+1} ; u_{1}(n+2), u_{j+1}(n+2)\right) \cdot<y, x_{1}, x_{2}, \ldots, x_{n+1}>_{j, n+2}
$$

(also see Notations (2-4) of Proposition 1 and (10)-(13) in Theorem 1) for every $y, x_{1}, \ldots, x_{n+1}$ in $G$. That is, $P_{n}$ is the two-sided $A$-module, where $A$ has the unit element.

By $R_{n}=R\left(P_{n}, M\right)$, we denote the family of all right homomorphisms of $P_{n}$ into $M$. For each $p \in R_{n}$, let an arbitrary element $\dot{p} \in C^{n}(A, M)$ in the additive group of all $n$ co-chains (that is, $n$ times $\mathcal{T}$-linear mappings of $A$ into $M)$ on $A$ with values in $M$ be prescribed by the formula $\dot{p}\left(a_{1}, \ldots, a_{n}\right)=$ $p\left(a_{1}, \ldots, a_{n}, 1\right)$ for all $a_{1}, \ldots, a_{n}$ in $A$. Consequently, $\left(\dot{p}\left(a_{1}, \ldots, a_{n}\right)\right) \cdot y=p\left(a_{1}, \ldots, a_{n}, y\right)$ for each $y \in A$, since $t_{n+3}\left(x_{1}, \ldots, x_{n+1}, 1, g ; l(n+3), u_{n+3}(n+3)\right)=1$ for all $x_{1}, \ldots, x_{n+1}$ and $g$ in $G$. This makes the mapping $p \mapsto \dot{p}$ an $\mathcal{T}$-linear isomorphism of $R_{n}$ onto $C^{n}(A, M)$.

Supply $C^{n}(A, M)$ with a two-sided $A$-module structure

(3) $\left(x_{0} \cdot f\right)\left(x_{1}, \ldots, x_{n}\right)=x_{0} \cdot\left(f\left(x_{1}, \ldots, x_{n}\right)\right)$ and

(4) $\left(f \cdot x_{0}\right)\left(x_{1}, . ., x_{n}\right)=\sum_{k=0}^{n-1}(-1)^{k} t_{n+1}\left(x_{0}, x_{1}, \ldots, x_{n} ; u_{1}(n+1), u_{k+2}(n+1)\right) \cdot f\left(x_{0}, \ldots, x_{k} x_{k+1}, \ldots, x_{n}\right)$ $+(-1)^{n}\left(f\left(x_{0}, \ldots, x_{n-1}\right)\right) \cdot x_{n}$

for each $f \in C^{n}(A, M)$ and all $x_{0}, x_{1}, \ldots, x_{n}$ in $G$, extending $f$ by $\mathcal{T}$-linearity on $A$ from $G$, where $u_{j}(n+$ 1) are given by Formulas (10)-(13) in Theorem 1. Thus, the mapping $p \mapsto \dot{p}$ is an operator isomorphism. Consequently, $H^{p}\left(A, R_{n}\right)$ is isomorphic with $H^{p}\left(A, C^{n}(A, M)\right)$ for each integer $(n$ and $p)$ such that $n \geq 0$ and $p \geq 0$. On the other hand, $H^{p}\left(A, C^{n}(A, M)\right)$ is isomorphic with $H^{p+n}(A, M)$ for each $p \geq 1$; hence, $H^{p}\left(A, R_{n}\right)$ is isomorphic with $H^{p+n}(A, M)$.

By virtue of Theorem 2 applied with $p=1$, we infer that $H^{n+1}(A, M)$ is isomorphic with the additive group of equivalence classes of right inessential enlargements of $M$ by $P_{n}$. 
Theorem 5. Let $M$ be a two-sided A-module, where $A$ is a nonassociative metagroup algebra over a commutative associative unital ring $\mathcal{T}$. Then, to each $n+1$-co-cycle $f \in Z^{n+1}(A, M)$, an enlargement of $M$ by a two-sided A-module $P_{n}$ corresponds such that $f$ becomes a co-boundary in it.

Proof. An $n+1$-co-cycle $f \in Z^{n+1}(A, M)$ induces an enlargement $(E, \eta)$ of $M$ by $P_{n}$ due to Theorem 4 . An element $h$ in $Z^{1}\left(A, R_{n}\right)$ corresponding to $f$ is characterized by the equality

$$
\left(h\left(x_{1}\right)\right)\left(x_{2}, \ldots, x_{n+1}, 1\right)=t_{n+1}\left(x_{1}, \ldots, x_{n+1} ; u_{1}(n+1), l(n+1)\right) \cdot f\left(x_{1}, \ldots, x_{n+1}\right)
$$

for all $x_{1}, \ldots, x_{n+1}$ in $G$. This enlargement $(E, \eta)$ as the two-sided $A$-module is $P_{n} \oplus M$ such that $x_{1} \cdot\left(\left(x_{2}, \ldots, x_{n+1}, 1\right), 0\right)=\left(x_{1} \cdot\left(x_{2}, \ldots, x_{n+1}\right), f\left(x_{1}, \ldots, x_{n+1}\right)\right)$. Let $\gamma\left(a_{1}, \ldots, a_{n}\right)=\left(a_{1}, \ldots, a_{n}, 0\right)$ for all $a_{1}, \ldots, a_{n}$ in $A$. Therefore, we deduce that $f\left(x_{1}, \ldots, x_{n+1}\right)=t_{n+1}\left(x_{1}, \ldots, x_{n+1} ; l(n+1), u_{1}(n+1)\right) \cdot\left\{x_{1}\right.$. $\left.\gamma\left(x_{2}, \ldots, x_{n+1}, 1\right)-\gamma\left(x_{1} \cdot\left(x_{2}, \ldots, x_{n+1}, 1\right)\right)\right\}$.

An $n$-co-chain $v \in C^{n}(A, E)$, defined by $v\left(a_{1}, \ldots, a_{n}\right)=\left(\left(a_{1}, \ldots, a_{n}, 1\right), 0\right)$, exists for all $a_{1}, \ldots, a_{n}$ in $A$. Thus, $f=\delta v$.

Theorem 6. Let $A$ be a nonassociative metagroup algebra over a commutative associative unital ring $\mathcal{T}$. Then, an algebra $B$ over $\mathcal{T}$ exists such that $B$ contains $A$ and each $\mathcal{T}$-homogeneous derivation $d: A \rightarrow A$ is the restriction of an inner derivation of $B$.

Proof. Naturally, an algebra $A$ has the structure of a two-sided $A$-module. In view of Theorem 1 , each derivation of the two-sided algebra $A$ can be considered an element of $Z^{1}(A, A)$.

Applying Theorem 5 by induction, one obtains a two-sided $A$-module $Q$ containing $M$ for which an arbitrary element of $Z^{n+1}(A, M)$ is represented as the co-boundary of an element of $C^{n}(A, Q)$. At the same time, $M$ and $Q$ satisfy Conditions (1)-(3) in Definition 3. This implies that the natural injection of $H^{n+1}(A, M)$ into $H^{n+1}(A, Q)$ maps $H^{n+1}(A, M)$ into zero.

Therefore, a two-sided $A$-module $E$ exists, which, as a two-sided $\mathcal{T}$-module, is a direct sum, $A \oplus P$, and $P$ is such that for each $f \in Z^{1}(A, A)$, an element $p \in P$ exists that generally depends on $f$ with the property $f(a)=a \cdot p-p \cdot a$. The metagroup $G$ corresponds to the algebra $A$. By enlarging $P$, if necessary, we can consider that to $P$, a metagroup $G$ also corresponds in such a manner that properties (1)-(3) in Definition 3 are fulfilled.

Now, we take $A \oplus P$ as the underlying two-sided $\mathcal{T}$-module of $B$ and supply it with the multiplication $\left(a_{1}, p_{1}\right)\left(a_{2}, p_{2}\right):=\left(a_{1} a_{2}, a_{1} \cdot p_{2}+p_{1} \cdot a_{2}\right)$ as the semidirect product for each $a_{1}, a_{2}$ in $A$ and $p_{1}, p_{2}$ in $P$. An embedding $\xi$ of $A$ into $B$ is $\xi(a)=(a, 0)$ for each $a$ in $A$. This implies that $f(a)=(a, 0)(0, p)-(0, p)(a, 0)=a(0, p)-(0, p) a$.

Theorem 7. Suppose that $A$ is a nonassociative metagroup algebra of finite order over a commutative associative unital ring $\mathcal{T}$ and $M$ is a finitely generated two-sided $A$-module. Then, $M$ is semisimple if and only if its cohomology group is null $H^{n}(A, M)=0$ for each natural number $n \geq 1$.

Proof. Certainly, if $E$ is an $A$-module and $N$ is its $A$-submodule, then a natural quotient morphism $\pi: E \rightarrow E / N$ exists. Therefore, an enlargement $(E, \eta)$ of a two-sided $A$-module $M$ by a two-sided $A$-module $N$ is inessential if and only if there is a submodule $T$ in $E$ complemented to $\xi(M)$ such that $T$ is isomorphic with $E / \xi(M)$, where $\xi$ is an embedding of $M$ into $E$. If $M$ is semisimple, then it is either a simple or a finite product of simple modules, since $M$ is finitely generated. For a finitely generated module $E$ and its submodule $N$, the quotient module $E / N$ is not isomorphic with $E$, since the algebra $A$ is of finite order over the commutative associative unital $\operatorname{ring} \mathcal{T}$.

By virtue of Theorems 4 and 5 , if, for an algebra $A$, its corresponding finitely generated two-sided $A$-modules are semisimple, then its cohomology groups of dimension $n \geq 1$ are zero.

Vice versa, suppose that $H^{n}(A, M)=0$ for each natural number $n \geq 1$. Consider a finitely generated two-sided $A$-module $E$ and its two-sided $A$-submodule $N$. At first, we take into account the right $A$-module structure $E_{r}$ of $E$ with the same right transformations, but with zero left transformations. Then, the left inessential $\left(E_{r}, \eta_{r}\right)$ enlargement of $M_{r}$ by $N_{r}=E_{r} / \xi_{r}\left(M_{r}\right)$ exists, where $\eta_{r}: E_{r} \rightarrow$ 
$E_{r} / \xi_{r}\left(M_{r}\right)$ is the quotient mapping and $\xi_{r}$ is an embedding of $M_{r}$ into $E_{r}$. From Theorem 4, it follows that the enlargement $\left(E_{r}, \eta_{r}\right)$ is right inessential. Analogously, considering the left $A$-module structures $E_{l}$ and $M_{l}$ we infer that $\left(E_{l}, \eta_{l}\right)$ is also left inessential.

Note 2. Let $A$ be a nonassociative metagroup algebra over a commutative associative unital ring $\mathcal{T}$ with a characteristic char $(\mathcal{T})$ other than two and three. Its opposite algebra $A^{\text {op }}$ exists. The latter, as an F-linear space, is the same, but with the multiplication $x \circ y=y x$ for each $x, y \in A^{o p}$. To each element $h \in A$ or $y \in A^{\text {op }}$, there is posed a left multiplication operator $L_{h}$ by the formula $x L_{h}=h x$ or a right multiplication operator $x R_{y}=x y$ for each $x \in A$, respectively. Having the anti-isomorphism operator $S: A \rightarrow A^{\text {op }}, A \ni x \mapsto x S \in A^{o p}$, $S(x y)=S(y) S(x)$, we get

(1) $R_{h} S=S L_{h S}$ and $L_{h} S=S R_{h S}$ for each $x$ and $h$ in A. Then, taking into account (1) analogously to formula (4) in Theorem 4 , we put $x_{0} \cdot\left(L_{x_{1}}, R_{x_{2}}\right)=\mathrm{t}_{3}^{-1}\left(x_{0}, x_{1}, x_{2}\right) \cdot\left(x_{0} L_{x_{1}}\right) L_{x_{2} S}-x_{0}\left(L_{x_{1}} R_{x_{2}}\right)$ $+\mathrm{t}_{3}^{-1}\left(x_{0}, x_{1}, x_{2}\right) \cdot\left(x_{0} R_{x_{1}}\right) R_{x_{2}}$.

Then, taking into account the multipliers $\mathrm{t}_{3}$, this gives

(2) $x_{0} \cdot\left(L_{x_{1}}, R_{x_{2}}\right)=x_{0}\left(L_{x_{1}} L_{x_{2} S}-L_{x_{1}} R_{x_{2}}+R_{x_{1} S} R_{x_{2}}\right)$ for all $x_{0}, x_{1}, x_{2}$ in $G$. Next, symmetrically, $S\left(x_{0} \cdot\right.$ $\left.\left(L_{x_{1}}, R_{x_{2}}\right)\right)$ provides the formula for $\left(L_{y_{1}}, R_{y_{2}}\right) \cdot y_{0}$ for each $y_{0}, y_{1}$ and $y_{2}$ in $G$. We consider the enveloping algebra $A^{e}=A \otimes_{\mathcal{T}} A^{o p}$. Extending these rules by $\mathcal{T}$-linearity on $A$ and $A \otimes_{\mathcal{T}} A^{o p}$ from $G$ one supplies the tensor product $M=A^{e}$ over $\mathcal{T}$ with the two-sided A-module structure.

Corollary 1. Let A be a semisimple, nonassociative metagroup algebra of finite order over a commutative associative unital ring $\mathcal{T}$ with a characteristic $\operatorname{char}(\mathcal{T})$ other than two and three, and let $M$ be a two-sided A-module described in Note 2. Then, $H^{n}(A, M)=0$ for each natural number $n \geq 1$.

Proof. Since $A$ is semisimple, then the module $M$ from Note 2 is semisimple. Consequently, the statement of this corollary follows from Theorem 7.

\section{Products of Metagroups}

The main subject of this paper are cohomologies on metagroups. Nonetheless, in this section, it is shortly demonstrated that there are abundant families of metagroups besides those which appear in areas described in the introduction.

Theorem 8. Let $G_{j}$ be a family of metagroups (see Definition 1 in Section 2), where $j \in J, J$ is a set. Then, their direct product $G=\prod_{j \in J} G_{j}$ is a metagroup and

(1) $\mathcal{C}(G)=\prod_{j \in J} \mathcal{C}\left(G_{j}\right)$

Proof. Each element $a \in G$ is written as $a=\left\{a_{j}: \forall j \in J, a_{j} \in G_{j}\right\}$. Therefore, a product $a b=$ $\left\{c: \forall j \in J, c_{j}=a_{j} b_{j}, a_{j} \in G_{j}, b_{j} \in G_{j}\right\}$ is a single-valued binary operation on $G$. Then, we get that $a \backslash b=\left\{d: \forall j \in J, d_{j}=a_{j} \backslash b_{j}, a_{j} \in G_{j}, b_{j} \in G_{j}\right\}$ and $a / b=\left\{d: \forall j \in J, d_{j}=a_{j} / b_{j}, a_{j} \in G_{j}, b_{j} \in G_{j}\right\}$. Moreover, $e_{G}=\left\{\forall j \in J, e_{G_{j}}\right\}$ is a neutral element in $G$, where $e_{G_{j}}$ denotes a neutral element in $G_{j}$ for each $j \in J$. Thus, Conditions (1)-(3) of Definition 1 in Section 2 are satisfied.

From Conditions (4)-(7) of Definition 1 in Section 2 for each $G_{j}$, we infer that

(2) $\operatorname{Com}(G):=\{a \in G: \forall b \in G, a b=b a\}=\left\{a \in G: a=\left\{a_{j}: \forall j \in J, a_{j} \in G_{j}\right\} ; \forall b \in G, b=\left\{b_{j}:\right.\right.$ $\left.\left.\forall j \in J, b_{j} \in G_{j}\right\} ; \forall j \in J, a_{j} b_{j}=b_{j} a_{j}\right\}=\prod_{j \in J} \operatorname{Com}\left(G_{j}\right)$,

(3) $N_{l}(G):=\{a \in G: \forall b \in G, \forall c \in G,(a b) c=a(b c)\}=\left\{a \in G: a=\left\{a_{j}: \forall j \in J, a_{j} \in G_{j}\right\} ; \forall b \in\right.$ $\left.G, b=\left\{b_{j}: \forall j \in J, b_{j} \in G_{j}\right\} ; \forall c \in G, c=\left\{c_{j}: \forall j \in J, c_{j} \in G_{j}\right\} ; \forall j \in J,\left(a_{j} b_{j}\right) c_{j}=a_{j}\left(b_{j} c_{j}\right)\right\}=$ $\prod_{j \in J} N_{l}\left(G_{j}\right)$, and similarly,

(4) $N_{m}(G)=\prod_{j \in J} N_{m}\left(G_{j}\right)$ and

(5) $N_{r}(G)=\prod_{j \in J} N_{r}\left(G_{j}\right)$.

This and (8) of Definition 1 in Section 2 imply that 
(6) $N(G)=\prod_{j \in J} N\left(G_{j}\right)$. Thus,

(7) $\mathcal{C}(G):=\operatorname{Com}(G) \cap N(G)=\prod_{j \in J} \mathcal{C}\left(G_{j}\right)$.

Let $a, b$, and $c$ be in $G$. Then,

$(a b) c=\left\{\left(a_{j} b_{j}\right) c_{j}: \forall j \in J, a_{j} \in G_{j}, b_{j} \in G_{j}, c_{j} \in G_{j}\right\}=\left\{\mathrm{t}_{3, G_{j}}\left(a_{j}, b_{j}, c_{j}\right) a_{j}\left(b_{j} c_{j}\right): \forall j \in J, a_{j} \in\right.$ $\left.G_{j}, b_{j} \in G_{j}, c_{j} \in G_{j}\right\}=\mathrm{t}_{3, G}(a, b, c) a(b c)$, where

(8) $\mathrm{t}_{3, G}(a, b, c)=\left\{\mathrm{t}_{3, G_{j}}\left(a_{j}, b_{j}, c_{j}\right): \forall j \in J, a_{j} \in G_{j}, b_{j} \in G_{j}, c_{j} \in G_{j}\right\}$.

Therefore, Formulas (7) and (8) imply that Condition (9) of Definition 1 in Section 2 is also satisfied. Thus, $G$ is a metagroup.

Remark 2. (1) Let $A$ and $B$ be two metagroups, and let $\mathcal{C}$ be a commutative group such that $\mathcal{C}_{m}(A) \hookrightarrow$ $\mathcal{C}, \mathcal{C}_{m}(B) \hookrightarrow \mathcal{C}, \mathcal{C} \hookrightarrow \mathcal{C}(A)$ and $\mathcal{C} \hookrightarrow \mathcal{C}(B)$, where $\mathcal{C}_{m}(A)$ denotes a minimal subgroup in $\mathcal{C}(A)$ generated by $\left\{t_{A}(a, b, c): a \in A, b \in A, c \in A\right\}$.

Using direct products, it is always possible to extend either $A$ or $B$ to get such a case. In particular, either $A$ or $B$ may be a group. Let an equivalence relation $\Xi$ on the Cartesian product $A \times B$ be such that

(2) $\quad(\gamma v, b) \Xi(v, \gamma b)$ and $(\gamma v, b) \Xi \gamma(v, b)$ and $(\gamma v, b) \Xi(v, b) \gamma$

for every $v$ in $A, b$ in $B$ and $\gamma$ in $\mathcal{C}$.

(3) Let $\phi: A \rightarrow \mathcal{A}(B)$ be a single-valued mapping, where $\mathcal{A}(B)$ denotes a family of all bijective surjective single-valued mappings of $B$ onto $B$ subjected to the conditions given below. If $a \in A$ and $b \in B$, then $b^{a}$ is written instead of $\phi(a) b$ for short, where $\phi(a): B \rightarrow B$. Also, let $\eta_{\phi}: A \times A \times B \rightarrow \mathcal{C}$, $\kappa_{\phi}: A \times B \times B \rightarrow \mathcal{C}$ and $\xi_{\phi}:((A \times B) / \Xi) \times((A \times B) / \Xi) \rightarrow \mathcal{C}$ be single-valued mappings written as $\eta, \kappa$, and $\xi$ for short, such that

(4) $\left(b^{u}\right)^{v}=b^{v u} \eta(v, u, b), e^{u}=e, b^{e}=b$;

(5) $\eta(v, u, \gamma b)=\eta(v, u, b)$;

(6) $(c b)^{u}=c^{u} b^{u} \kappa(u, c, b)$;

(7) $\kappa(u, \gamma c, b)=\kappa(u, c, \gamma b)=\kappa(u, c, b)$ and

$\kappa(u, \gamma, b)=\kappa(u, b, \gamma)=e ;$

(8) $\xi((\gamma u, c),(v, b))=\xi((u, c),(\gamma v, b))=\xi((u, c),(v, b))$ and

$\xi((\gamma, e),(v, b))=e$ and $\xi((u, c),(\gamma, e))=e$

for every $u$ and $v$ in $A, b, c$ in $B, \gamma$ in $\mathcal{C}$, where e denotes the neutral element in $\mathcal{C}$ and in $A$ and $B$.

We put

(9) $\left(a_{1}, b_{1}\right)\left(a_{2}, b_{2}\right)=\left(a_{1} a_{2}, \xi\left(\left(a_{1}, b_{1}\right),\left(a_{2}, b_{2}\right)\right) b_{1} b_{2}^{a_{1}}\right)$

for each $a_{1}, a_{2}$ in $A, b_{1}$ and $b_{2}$ in $B$.

The Cartesian product $A \times B$ supplied with such a binary operation (9) is denoted by $A \otimes^{\phi, \eta, \kappa, \tau} B$.

Theorem 9. Let the conditions of Remark 2 be fulfilled. Then, the Cartesian product $A \times B$ supplied with a binary Operation (9) in Remark 2 is a metagroup.

Proof. From the conditions of Remark 2, it follows that the binary operation (9) in Remark 2 is single-valued.

Let $I_{1}=\left(\left(a_{1}, b_{1}\right)\left(a_{2}, b_{2}\right)\right)\left(a_{3}, b_{3}\right)$ and $I_{2}=\left(a_{1}, b_{1}\right)\left(\left(a_{2}, b_{2}\right)\left(a_{3}, b_{3}\right)\right)$, where $a_{1}, a_{2}, a_{3}$ belong to $A$, and $b_{1}, b_{2}, b_{3}$ belong to $B$. Then, we infer that

$I_{1}=\left(\left(a_{1} a_{2}\right) a_{3}, \xi\left(\left(a_{1}, b_{1}\right),\left(a_{2}, b_{2}\right)\right) \xi\left(\left(a_{1} a_{2}, b_{1} b_{2}^{a_{1}}\right),\left(a_{3}, b_{3}\right)\right)\left(b_{1} b_{2}^{a_{1}}\right) b_{3}^{a_{1} a_{2}}\right)$ and

$I_{2}=\left(a_{1}\left(a_{2} a_{3}\right), \xi\left(\left(a_{1}, b_{1}\right),\left(a_{2} a_{3}, b_{2} b_{3}^{a_{2}}\right)\right)\left[\xi\left(\left(a_{2}, b_{2}\right),\left(a_{3}, b_{3}\right)\right)\right]^{a_{1}}\right.$

$\left.b_{1}\left(b_{2}^{a_{1}} b_{3}^{a_{1} a_{2}}\right) \kappa\left(a_{1}, b_{2}, b_{3}^{a_{2}}\right) \eta\left(a_{1}, a_{2}, b_{3}\right)\right)$. Therefore,

(1) $I_{1}=\mathrm{t}_{3}\left(\left(a_{1}, b_{1}\right),\left(a_{2}, b_{2}\right),\left(a_{3}, b_{3}\right)\right) I_{2}$ with

(2) $\mathrm{t}_{3}\left(\left(a_{1}, b_{1}\right),\left(a_{2}, b_{2}\right),\left(a_{3}, b_{3}\right)\right)=\mathrm{t}_{3, A}\left(a_{1}, a_{2}, a_{3}\right) \mathrm{t}_{3, B}\left(b_{1}, b_{2}^{a_{1}}, b_{3}^{a_{1} a_{2}}\right)$ 


$$
\begin{aligned}
& \xi\left(\left(a_{1}, b_{1}\right),\left(a_{2} a_{3}, b_{2} b_{3}^{a_{2}}\right)\right)\left[\xi\left(\left(a_{2}, b_{2}\right),\left(a_{3}, b_{3}\right)\right)\right]^{a_{1}} \kappa\left(a_{1}, b_{2}, b_{3}^{a_{2}}\right) \eta\left(a_{1}, a_{2}, b_{3}\right) \\
& /\left[\xi\left(\left(a_{1}, b_{1}\right),\left(a_{2}, b_{2}\right)\right) \xi\left(\left(a_{1} a_{2}, b_{1} b_{2}^{a_{1}}\right),\left(a_{3}, b_{3}\right)\right)\right] .
\end{aligned}
$$

Apparently, $\mathrm{t}_{3, A \otimes \phi, \eta, x, \xi_{B}}\left(\left(a_{1}, b_{1}\right),\left(a_{2}, b_{2}\right),\left(a_{3}, b_{3}\right)\right) \in \mathcal{C}$ for each $a_{j} \in A, b_{j} \in B, j \in\{1,2,3\}$, where for shortening of a notation, $\mathrm{t}_{3, A \otimes \otimes^{\phi, \eta, \kappa, \tilde{\xi}} B}$ is denoted by $\mathrm{t}_{3}$.

If $\gamma \in \mathcal{C}$, then

$\gamma\left(\left(a_{1}, b_{1}\right)\left(a_{2}, b_{2}\right)\right)=\left(\gamma a_{1} a_{2}, \xi\left(\left(a_{1}, b_{1}\right),\left(a_{2}, b_{2}\right)\right) b_{1} b_{2}^{a_{1}}\right)=\left(a_{1} a_{2}, b_{1} b_{2}^{a_{1}}\right) \gamma \xi\left(\left(a_{1}, b_{1}\right),\left(a_{2}, b_{2}\right)\right)=$ $\left(\left(a_{1}, b_{1}\right)\left(a_{2}, b_{2}\right)\right) \gamma$.

Hence, $\gamma \in \mathcal{C}\left(A \otimes^{\phi, \eta, \kappa, \xi} B\right)$. Consequently, $\mathcal{C} \subseteq \mathcal{C}\left(A \otimes^{\phi, \eta, \kappa, \xi} B\right)$.

Next, we consider the following equation:

(3) $\left(a_{1}, b_{1}\right)(a, b)=(e, e)$, where $a \in A, b \in B$.

From (2) of Definition 1 in Section 2 and (9) in Remark 2, we deduce that

(4) $a_{1}=e / a$.

Consequently, $\xi\left(\left(e / a, b_{1}\right),(a, b)\right) b_{1} b^{(e / a)}=e$, and hence

(5) $b_{1}=e /\left[\xi\left(\left(e / a, b^{(e / a)}\right),(a, b)\right) b^{(e / a)}\right]$.

Thus, $a_{1} \in A$ and $b_{1} \in B$ given by (4) and (5) provide a unique solution of (3).

Similarly, from the following equation

(6) $(a, b)\left(a_{2}, b_{2}\right)=(e, e)$, where $a \in A, b \in B$ we infer that

(7) $a_{2}=a \backslash e$.

Consequently, $\xi\left((a, b),\left(a \backslash e, b_{2}\right)\right) b b_{2}^{a}=e$, and hence, $b_{2}^{a}=\left[\xi\left((a, b),\left(a \backslash e, b_{2}\right)\right) b\right] \backslash e$. On the other hand, $\left(b_{2}^{a}\right)^{e / a}=\eta\left(e / a, a, b_{2}\right) b_{2}$ Consequently,

(8) $\quad b_{2}=(b \backslash e)^{e / a} /\left\{\left[\left(\xi\left((a, b),\left(a \backslash e,(b \backslash e)^{e / a}\right)\right)\right]^{e / a} \eta\left(e / a, a,(b \backslash e)^{e / a}\right)\right\}\right.$.

Thus, Formulas (7) and (8) provide a unique solution to (6).

Next, we put $\left(a_{1}, b_{1}\right)=(e, e) /(a, b)$ and $\left(a_{2}, b_{2}\right)=(a, b) \backslash(e, e)$ and

(9) $\quad(a, b) \backslash(c, d)=((a, b) \backslash(e, e))(c, d)$

(10) $\quad(c, d) /(a, b)=(c, d)((e, e) /(a, b))$

$\mathrm{t}_{3}((e, e) /(a, b),(a, b),(a, b) \backslash(e, e)) / \mathrm{t}_{3}((c, d)(e /(a, b)),(a, b),(a, b) \backslash(e, e))$

and $e_{G}=(e, e)$, where $G=A \otimes^{\phi, \eta, \kappa, \xi} B$. Note that (3) of Definition 1 in Section 2 follows on from (8) and (9) of Remark 2. Therefore, Properties (1)-(3) and (9) of Definition 1 in Section 2 are fulfilled for $A \otimes \otimes^{\phi, \eta, \kappa, \xi} B$.

Definition 5. The metagroup $A \otimes^{\phi, \eta, \kappa, \xi} B$ provided by Theorem 9 is called a smashed product of metagroups $A$ and $B$ with smashing factors $\phi, \eta, \kappa$, and $\xi$.

Remark 3. From Theorems 8 and 9, it follows that by taking the nontrivial mappings $\eta, \kappa$, and $\xi$ and starting from groups with nontrivial $\mathrm{C}\left(G_{j}\right)$ or $\mathrm{C}(A)$, it is possible to construct new metagroups with nontrivial $\mathrm{C}(G)$, and ranges $\mathrm{t}_{3, G}(G, G, G)$ of $\mathrm{t}_{3, G}$ may be infinite. Indeed, using extensions of groups (or metagroups) by semidirect or direct products, it is possible to take initial groups (or metagroups) $A$ and $B$ such that quotient groups $A_{1}$ of $A$ by $C(A)$ and $B_{1}$ of $B$ by $C(B)$ are infinite. Therefore, their automorphism groups $A u t\left(A_{1}\right)$ and $A u t\left(B_{1}\right)$ are infinite, because they contain all inner automorphisms.

With suitable smashing factors $\phi, \eta, \kappa$, and $\xi$ and with nontrivial metagroups or groups $A$ and $B$ it is easy to get examples of metagroups in which $e / a \neq a \backslash e$ for an infinite family of elements $a$ in $A \otimes^{\phi, \eta, \kappa, \xi} B$ using Formulas (1) in Lemma 2 in Section 2 and (2) in Theorem 9. Evidently smashed products (see Remark 2 and Theorem 9) are nonassociative generalizations of semidirect products. 
Smashed twisted products and smashed twisted wreath products of metagroups or groups are described in [34]. They also provide tools for a construction of a wide class of metagroups and nonassociative algebras with metagroup relations.

Conclusions 1. The results of this article can be used for further studies of cohomology theory of nonassociative algebras and noncommutative manifolds with metagroup relations. It is interesting to mention possible applications in mathematical coding theory, analysis of information flows, and their technical realizations [35-38], because codes are frequently based on binary systems and algebras. Indeed, metagroup relations are weaker than relations in groups. Therefore, a code complexity can increase by using nonassociative algebras with metagroup relations in comparison with group algebras or Lie algebras.

Besides the applications of cohomologies outlined in the introduction, they also can be used in mathematical physics and quantum field theory. This also can be applied to cohomologies of PDEs and solutions of PDEs with boundary conditions, which can have practical importance [27,39]. It will be interesting to investigate cohomologies of nonassociative algebras related with a class of directed ringoids $[40,41]$, because the latter have applications to non locally compact groups.

Funding: This research received no external funding.

Conflicts of Interest: The author declares no conflict of interest.

\section{References}

1. Cartan, H.; Eilenberg, S. Homological Algebra; Princeton University Press: Princeton, NJ, USA, 1956.

2. Goto, M.; Grosshans, F.D. Semisimple Lie algebras; Marcel Dekker, Inc.: New York, NY, USA, 1978.

3. Shang, Y. A Lie algebra approach to susceptible-infected-susceptible epidemics. Electron. J. Differ. Equat. 2012, 2012, 1-7.

4. Shang, Y. Analytic solution for an in-host viral invection model with time-inhomogeneous rates. Acta Phys. Pol. 2015, 46, 1567-1577. [CrossRef]

5. Shang, Y. Lie algebraic discussion for affinity based information diffusion in social networks. Open Phys. 2017, 15, 705-711. [CrossRef]

6. Allcock, D. Reflection groups and octave hyperbolic plane. J. Algebra 1998, 213, 467-498. [CrossRef]

7. Baez, J.C. The octonions. Bull. Am. Math. Soc. 2002, 39, 145-205. [CrossRef]

8. Dickson, L.E. The Collected Mathematical Papers; Chelsea Publishing Co.: New York, NY, USA, 1975; Volumes 1-5.

9. Kantor, I.L.; Solodovnikov, A.S. Hypercomplex Numbers; Springer: Berlin, Germany, 1989.

10. Schafer, R.D. An Introduction to Nonassociative Algebras; Academic Press: New York, NY, USA, 1966.

11. Serôdio, R. On octonionic polynomials. Adv. Appl. Clifford Algebras 2007, 17, 245-258.

12. Frenod, E.; Ludkowski, S.V. Integral operator approach over octonions to solution of nonlinear PDE. Far East J. Math. Sci. (FJMS) 2018, 103, 831-876. [CrossRef]

13. Girard, P.R. Quaternions, Clifford Algebras and Relativistic Physics; Birkhäuser: Basel, Switzerland, 2007.

14. Gürlebeck, K.; Sprössig, W. Quaternionic and Clifford Calculus for Physicists and Engineers; John Wiley and Sons: Chichester, NH, USA, 1997.

15. Gürsey, F.; Tze, C.-H. On the Role of Division, Jordan and Related Algebras in Particle Physics; World Scientific Publishing Co.: Singapore, 1996.

16. Krausshar, R.S. Generalized Analytic Automorphic Forms in Hypercomplex Spaces; Birkhäuser: Basel, Switzerland, 2004.

17. Ludkovsky, S.V. Wrap groups of connected fiber bundles: Their structure and cohomologies. Lie Groups: New Research; Canterra, A.B., Ed.; Nova Science Publishers, Inc.: New York, NY, USA, 2009; pp. 53-128.

18. Ludkowski, S.V. Decompositions of PDE over Cayley-Dickson algebras. Rend. Istit. Mat. Univ. 2014, 46, 1-23.

19. Ludkowski, S.V. Integration of vector Sobolev type PDE over octonions. Complex Var. Elliptic Equat. 2016, 61, 1014-1035.

20. Ludkowski, S.V. Manifolds over Cayley-Dickson algebras and their immersions. Rend. Istit. Mat. Univ. 2013, $45,11-22$. 
21. Ludkovsky, S.V. Normal families of functions and groups of pseudoconformal diffeomorphisms of quaternion and octonion variables. J. Math. Sci. N. Y. 2008, 150, 2224-2287. [CrossRef]

22. Ludkovsky, S.V. Functions of several Cayley-Dickson variables and manifolds over them. J. Math. Sci. N. Y. 2007, 141, 1299-1330. [CrossRef]

23. Ludkovsky, S.V.; Sprössig, W. Ordered representations of normal and super-differential operators in quaternion and octonion Hilbert spaces. Adv. Appl. Clifford Alg. 2010, 20, 321-342.

24. Ludkovsky, S.V.; Sprössig, W. Spectral theory of super-differential operators of quaternion and octonion variables. Adv. Appl. Clifford Alg. 2011, 21, 165-191.

25. Ludkovsky, S.V. Integration of vector hydrodynamical partial differential equations over octonions. Complex Var. Elliptic Equat. 2013, 58, 579-609. [CrossRef]

26. Ludkowski, S.V. Automorphisms and derivations of nonassociative $C^{*}$ algebras. Linear Multilinear Algebra 2018, 1-8. [CrossRef]

27. Pommaret, J.F. Systems of Partial Differential Equations and Lie Pseudogroups; Gordon and Breach Science Publishers: New York, NY, USA, 1978.

28. Bourbaki, N. Algèbre. In Algèbre Homologique; Springer: Berlin, Germany, 2007.

29. Bredon, G.E. Sheaf Theory; McGarw-Hill: New York, NY, USA, 2012.

30. Hochschild, G. On the cohomology theory for associative algebras. Ann. Math. 1946, 47, 568-579. [CrossRef]

31. Chapoton, F.; Livernet, M. Pre-Lie algebras and rooted trees operad. Int. Math. Res. Not. 2001, 8, 395-408. [CrossRef]

32. Dzhumadil'daev, A.; Zusmanovich, P. The alternative operad is not Koszul. Exper. Math. 2011, 20, 138-144. [CrossRef]

33. Remm, E.; Goze, M. A class of nonassociative algebras including flexible and alternative algebras, operads and deformations. J. Gener. Lie Theory Appl. 2015, 9, 1-6.

34. Ludkowski, S.V. Metagroups and their smashed twisted wreath products. arXiv 2018, arXiv:1809.02801.

35. Blahut, R.E. Algebraic Codes for Data Transmission; Cambridge University Press: Cambridge, UK, 2003.

36. Magomedov, S.G. Assessment of the impact of confounding factors in the performance information security. Rus. Technol. J. 2017, 5, 47-56.

37. Sigov, A.S.; Andrianova, E.G.; Zhukov, D.O.; Zykov, S.V.; Tarasov, I.E. Quantum informatics: Overview of the main achievements. Rus. Technol. J. 2019, 7, 5-37. [CrossRef]

38. Shum, K.P.; Ren, X.; Wang, Y. Semigroups on semilattice and the constructions of generalized cryptogroups. Southeast Asian Bull. Math. 2014, 38, 719-730.

39. Zaikin, B.A.; Bogadarov, A.Y..; Kotov, A.F.; Poponov, P.V. Evaluation of coordinates of air target in a two-position range measurement radar. Rus. Technol. J. 2016, 4, 65-72.

40. Ludkovsky, S.V. On transfinite construction of a class of directed topological ringoids. JP J. Algebra Number Theory Appl. 2015, 37, 185-208. [CrossRef]

41. Ludkowski, S.V. Skew continuous morphisms of ordered lattice ringoids. Mathematics 2016, 4, 17. [CrossRef]

(C) 2019 by the authors. Licensee MDPI, Basel, Switzerland. This article is an open access article distributed under the terms and conditions of the Creative Commons Attribution (CC BY) license (http://creativecommons.org/licenses/by/4.0/). 